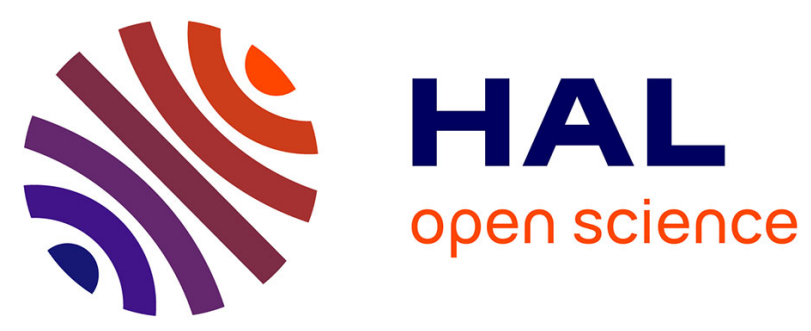

\title{
A value for bi-cooperative games
}

Christophe Labreuche, Michel Grabisch

\section{To cite this version:}

Christophe Labreuche, Michel Grabisch. A value for bi-cooperative games. International Journal of Game Theory, 2008, 37 (3), pp.409-438. 10.1007/s00182-008-0126-5 . halshs-00308738

\section{HAL Id: halshs-00308738 https://shs.hal.science/halshs-00308738}

Submitted on 16 Jan 2009

HAL is a multi-disciplinary open access archive for the deposit and dissemination of scientific research documents, whether they are published or not. The documents may come from teaching and research institutions in France or abroad, or from public or private research centers.
L'archive ouverte pluridisciplinaire HAL, est destinée au dépôt et à la diffusion de documents scientifiques de niveau recherche, publiés ou non, émanant des établissements d'enseignement et de recherche français ou étrangers, des laboratoires publics ou privés. 


\title{
A value for bi-cooperative games
}

\author{
Christophe Labreuche $^{1}$ and Michel Grabisch ${ }^{2}$
}

1. THALES Research and Technology France, RD 128, 91767 Palaiseau Cedex, France

2. University of Paris I, CERMSEM - 106-112, Bd de l'Hôpital,75647 Paris Cedex 13, France

\begin{abstract}
Bi-cooperative games have been introduced by Bilbao et al as a generalization of TU cooperative games, where each player can participate positively to the game, negatively, or do not participate. In this paper, we propose a definition of a share of the wealth obtained by some players after they decided on their participation to the game. It turns out that the cost allocation rule does not look for a given player to her contribution at the opposite action to the one she chooses. The relevance of the value is discussed on several examples.
\end{abstract}

Keywords: bi-cooperative games, value, efficiency.

\section{Introduction}

Acquisition and possession of scarce resource is economically costly so that the mutual use of such resource by several agents or organizations usually implies positive externalities on these latters. Once the agents have decided to cooperate, the question of how to allocate the use of the resource as well as how to share its costs and benefits among the users arises. The main concern of Cooperative Game Theory is precisely to deal with this issue. The most classical model is the concept of game in characteristic form. When all side payments are possible among players, this leads to usual TU games. This representation is used to model the exchange of private goods under private ownership, or the collaborative use of commons that can be a public good or a common technology. This has been applied to very different areas such as water supply, power supply, distributive justice, or the share of maintenance costs among the user of a common shared railway infrastructure $[10,17,11]$.

TU games are encoded with a function $v: 2^{N} \rightarrow \mathbb{R}$ where $N$ is the set of players, which assigns to any coalition $S \subseteq N$ its worth $v(S)$ representing 
the stand alone worth of coalition $S$, e.g. the cost for supplying all players of $S$ with the resource. In the microeconomic situations modeled by TU games, all players participating to the game have usually the same role. For instance, all players can be consumers of the commons.

As shown in the following example, there are situations in which the players can choose between two different roles.

Example 1 (Irrigation Network) Water is a critical resource in many agricultural areas, especially in deserts. Farmers have thus interest in cooperating and constructing common irrigation networks. We assume here that there is only one possible location for the setting up of a well in a given area. Hence the farmers who possess a parcel close to that well must share this well and decide to construct jointly an irrigation network that will convey water to their parcel.

We consider an example with three farmers. The situation of the parcels is given in Figure 1.

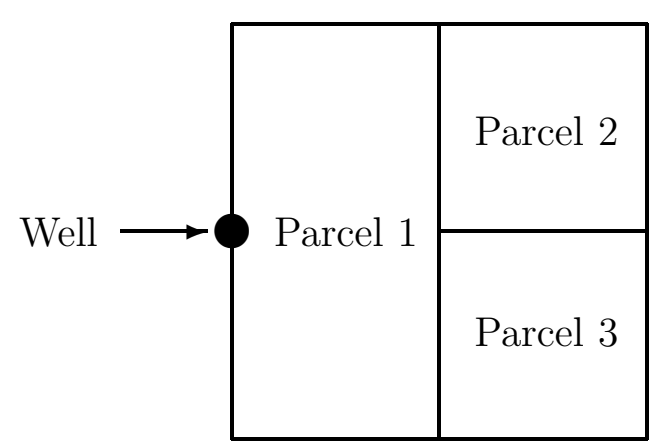

Fig. 1 Situation of the three parcels and the well .

The water network basically consists of pipes that are buried at the borders of the parcels. The parcel of the first farmer is located just closeby the well, and is also the largest one. To supply water to the two other parcels, it is necessary to skirt around farmer 1's parcel. This requires a long distance of pipes and leads thus a huge cost. Figure 2 below presents the different paths of the network in all possible cases: Case $a$ corresponds to the situation where only farmer 1 asks for water, case $b$ to the situation where farmer 2 asks for water and not farmer 3, case $c$ to the situation where farmer 3 asks for water and not farmer 2 , and case $d$ to the situation where farmers 2 and 3 both ask for water. Figure 2 also gives the cost in each situation. The cost of digging the well is 1 and the cost of buying and burying the pipes is 1 per unit of length, where the union of the three parcels corresponds to a square of $10 \times 10$ in unit of length.

The cost for constructing the network supplying water to all possible coalitions of farmers defines a usual TU game which is a tree game [10]. 


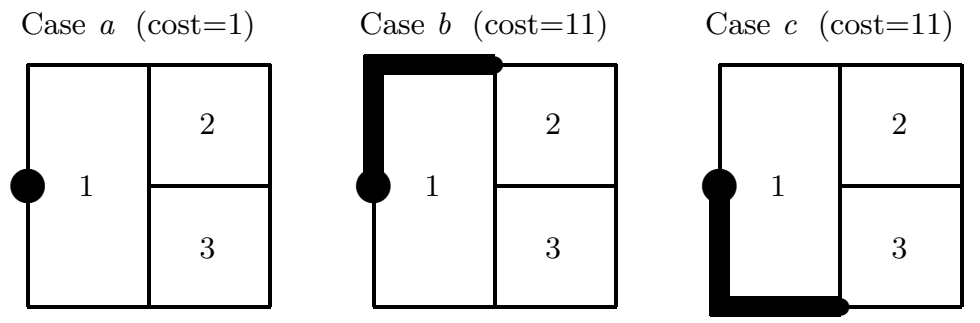

Case $d \quad(\operatorname{cost}=16)$

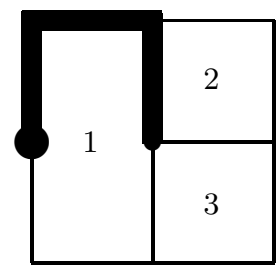

Fig. 2 The four different cases of irrigation networks.

Since parcel 1 has an important surface, the price of supplying water to parcels 2 and 3 is very large. An option to reduce hugely the cost is to decide that the pipes cut through farmer 1's parcel in its middle. This leads to the situation depicted in Figure 3. Yet, the decision of cutting parcel 1's field can only be made by farmer 1 since this causes annoyance to her. It will indeed implies a decrease of the estate price, and a higher complexity of the sweeping of the parcel during ploughing. Therefore, farmer 1 wants to quantify the consequence of the two possible options (allowing or not trespassing) before making her final decision. Remark that farmers 2 and 3

Case $e \quad(\operatorname{cost}=6)$

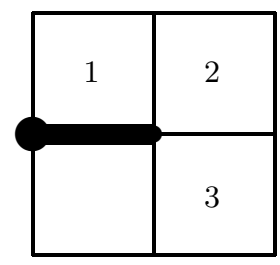

Fig. 3 The case of parcel 1 being cut in its middle.

may also allow the pipes to cut their field but this will not help in reducing the length of the network, and thus the overall cost.

In previous example, farmer 1 when asking for water can adopt two attitudes - namely either requiring that the pipes lie on the border of her parcel (first attitude), or allowing the pipes to cut her field (second attitude). 
The cost of the irrigation network increases when a farmer switches from not asking water to asking water while adopting the first attitude (e.g. from 11 up to 16 for farmer 2 if the other two farmers already asked water). Moreover, the network cost usually decreases when farmer 1 switches from not asking water to asking water while adopting the second attitude (e.g. from 16 down to 6 if the other two farmers already asked water).

More generally, one sees that there exists situations in which the players can choose among two roles or attitudes - called hereafter positive and negative roles - when deciding to cooperate, such that the choice of one of the two roles implies opposite externalities on the other players. The worth increases when a player decides to participate to the game through the positive role, compare to the case where this player does not participate to the game. Symmetrically, the worth generally decreases when a player decides to participate to the game through the negative role. A player that decides to cooperate with the positive role is called positive contributor, and a player that decides to cooperate with the negative role is called negative contributor. More examples of this situation are given in Section 2.

There exist TU games in which the participants can take two opposite roles. This is the case of market games in which some players are potential sellers that own several items of commodities, and the other players are buyers who want to possess some commodities. However, in these games, the role of the players cannot vary since it is fixed in advance. Moreover, $v(S)$ represents the largest social wealth players of $S$ can produce after bargaining among themselves. Clearly $v$ is monotone since the more players in the coalition the larger the wealth of the coalition. Thus, the situation is completely different from that depicted in Example 1.

It can be assumed that the role of each player when participating to the game is given exogenously and can thus be frozen. Hence each player can either cooperate given her exogenous choice of cooperation, or not participate to the game, which can be translated into a TU game. However, unlike market games where a buyer cannot become a seller and vice versa, a positive contributor could have decided to be a negative contributor. Computing the share alloted to each player from previous TU game implies that the contribution of a player when she chooses the opposite behavior (positive vs. negative role) to that given exogenously, is ruled out in the share. Since players may hesitate between two roles (see farmer 1 in Example 1), there is a priori no argument for discarding the opposite role in the computation of the sharing rule. This shows that the TU model is not sufficient. One needs to know the role chosen by the active players. Thus two disjoint coalitions are necessary - one for the positive contributors and one for the negative ones. The game we need is thus a function that assigns to any couple of disjoint coalitions a wealth. This corresponds to bi-cooperative games [3].

A central question in Cooperative Game Theory is how the players should share the total wealth $v(N)$ of a TU game $v$ if the grand coalition $N$ forms. A solution is a payoff vector $x \in \mathbb{R}_{+}^{N}$ such that $\sum_{i \in N} x_{i}=v(N)$. 
As an example, the Shapley value is a solution concept that evaluates the players' mean prospect.

Three different definitions of a value have been proposed for bi-cooperative games $[5,7,2]$. Beyond the question of the choice of the best suited value among the three previous ones, the interpretation of the notion of solution for a bi-cooperative game is not so obvious. These values satisfy the same efficiency axiom, which states that the value is a share of the difference of worth between situations where all players are positive contributors and where all players are negative contributors. This axiom is technical and lacks of clear interpretation. As a result, the solutions defined in $[5,7,2]$ cannot be viewed as a share of the wealth of the players but represents a mean prospect over the three possible choices of all players (i.e. being positive or negative contributor, or do not participate).

The goal of this paper is to revisit this problem. We abandon the idea of a value that represents a mean prospect whatever the level of participation of the players to the game. What we aim to do is similar to what is done in cost allocation problems in which the sought value is a share of the total cost corresponding to the actual demand asked by each player. It is thus natural to define, in our case, efficiency as the property such that the payoff is a share of the worth obtained by the players after they have decided either to be positive contributor, negative contributor or absent (see Section 3). Five other axioms are used to characterize a value for bi-cooperative games: linearity, null player, monotonicity and two symmetry axioms. An important result is obtained in Section 3. The main result of this paper proves that under linearity, null player, monotonicity and efficiency axioms, the payoff for a positive contributor depends only on her added-value from absent to positive contributor. The information regarding how this player behaves when she becomes negative contributor is not relevant. The same argument holds symmetrically for negative contributors. It is not possible to define an allocation rule that looks at the contribution for the opposite action to the one chosen by each agent (positive vs. negative). Hence for the computation of the value, one can restrict the bi-cooperative game to a usual TU game. Even though there is much more information contained in the bi-cooperative game than in the restricted game, the remaining terms cannot be used in a coherent way. The Shapley value is then applied to the TU game so obtained.

An overall value that is not conditional on the choice of the action of each player is defined in Section 4. It is an expected value computed from the value defined earlier. The expression we obtain is different from the existing ones $[5,7,2]$.

Section 5 discusses on the relevance of our value on several examples. Section 6 compares our proposal to previous ones.

In this paper, we have assumed that the choice of each player to be positive or negative contributor, or to be absent is exogenous. However, the bi-cooperative model can be seen as an intermediate argument towards the 
strategic choice of the best action for each player. This view is sketched in the conclusion (Section 7).

\section{Bi-cooperative games: definition and examples}

Throughout the paper, $N:=\{1, \ldots, n\}$ will denote the finite set of players. Cardinalities of coalitions will be denoted by corresponding lower case letters, i.e., $|S|=: s$.

Formally, we introduce $\mathcal{Q}(N):=\{(S, T) \mid S, T \subseteq N, S \cap T=\emptyset\}$, the set of pairs of disjoint coalitions. A bi-cooperative game is a function $v$ : $\mathcal{Q}(N) \longrightarrow \mathbb{R}$ with $v(\emptyset, \emptyset)=0$, where $v(S, T)$ is the worth when players in $S$ are positive contributors, players in $T$ are negative contributors, and the remaining players do not participate. Since cooperative games focus on the added-value of players when they participate to the game, it is natural that the worth $v(\emptyset, \emptyset)$ when no player participates to the game vanishes. In the context of cost sharing problems, condition $v(\emptyset, \emptyset)=0$ means that the cost when no player demands the technology or the commons is zero. We denote by $\mathcal{G}^{[2]}(N)$ the set of all bi-cooperative games on $N$.

A ternary voting game is a particular bi-cooperative game where $v$ can take only two values, value 1 when the bill is accepted and value 0 when the bill is rejected [5]. Worth $v(S, T)$ corresponds to the result of the vote when $S$ are the voters in favor of the bill, $T$ are the voters against the bill, and the remaining voters are abstentionist. Abstention is an alternative option to the usual yes and no opinions.

Example 2 (Irrigation Network continued) We are now in position to construct the bi-cooperative game of Example 1 from the costs given in Figures 2 and 3.

$$
\begin{aligned}
& v(\emptyset, \emptyset)=0 \quad, \quad v(\{1\}, \emptyset)=v(\emptyset,\{1\})=1 \quad(\text { case } a) \\
& v(\{2\}, \emptyset)=v(\{3\}, \emptyset)=v(\{1,2\}, \emptyset)=v(\{1,3\}, \emptyset)=11 \quad(\text { cases } b \text { and } c) \\
& v(\{2,3\}, \emptyset)=v(\{1,2,3\}, \emptyset)=16 \quad(\text { case } d) \\
& v(\{2\},\{1\})=v(\{3\},\{1\})=v(\{2,3\},\{1\})=6 \quad(\text { case } e)
\end{aligned}
$$

and for any $(S, T) \in \mathcal{Q}(\{2,3\})$

$$
\begin{aligned}
& v(S \cup\{1\}, T)=v(S \cup T \cup\{1\}, \emptyset) \\
& v(S, T)=v(S \cup T, \emptyset) \\
& v(S, T \cup\{1\})=v(S \cup T,\{1\})
\end{aligned}
$$

which means choosing positive or negative roles for farmers 2 and 3 has no consequence on the overall cost. 
A player $i$ is called positively monotone if

$$
\forall(S, T) \in \mathcal{Q}(N \backslash\{i\}) \quad v(S \cup\{i\}, T) \geq v(S, T) .
$$

In Example 1, all farmers are clearly positively monotone. A player $i$ is called negatively monotone if

$$
\forall(S, T) \in \mathcal{Q}(N \backslash\{i\}) \quad v(S, T \cup\{i\}) \leq v(S, T) .
$$

In Example 1, farmer 1 is negatively monotone, except for bi-coalition $(\emptyset, \emptyset)$. A bi-cooperative game is said to be monotone if all players are positively and negatively monotone. Bi-cooperative games which are monotone have been introduced independently by the authors under the name of bi-capacities $[6,14]$.

When

$$
\forall(S, T) \in \mathcal{Q}(N \backslash\{i\}) \quad v(S \cup\{i\}, T)=v(S, T \cup\{i\})
$$

the choice of the positive or negative role for player $i$ has no impact on the added value of this player when joining a coalition. Such player is called symmetric contributor. In Example 1, farmers 2 and 3 are symmetric contributors.

For illustrative purpose, we present three other examples of bi-cooperative games.

Example 3 (Urban Water Supply) Three cities want to share the cost of a common water supply network. In the original project, the water is supplied by a unique factory $F$ which pumps in the groundwater aquifer. The geographical situation of the cities, the factory and the water network (in dotted lines) is depicted in Figure 4. The problem is to share the water supply costs. The cost of conveying water to a city depends mainly on its distance to $F$ and its altitude, since this has an impact on the number of intermediate raising pumps that shall be put on the network. If the net is private and constructed by the cities, the share of cost will be based on the effective part of each city in the overall cost.

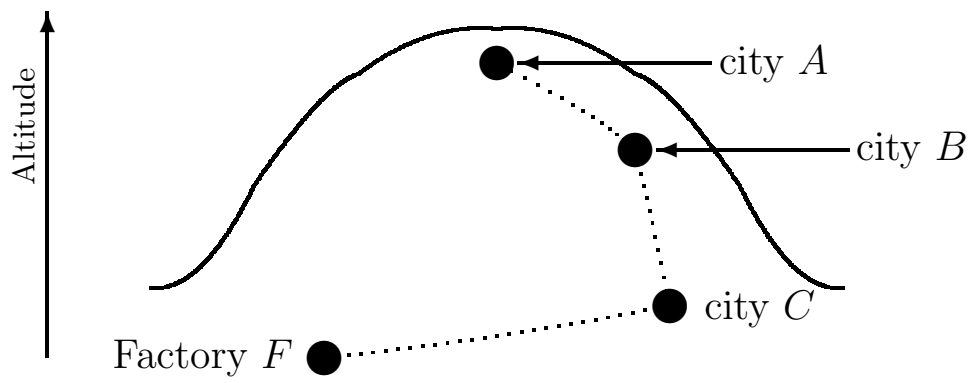

Fig. 4 Situation of the three cities (the vertical axis indicates altitude). 
City $C$ is a large city located in the valley. The price for supplying only $C$ is $p_{C}$. Cities $A$ and $B$ are small cities located on the hill. The price of providing water uphill is quite expensive. We denote by $p_{A}, p_{B}$ and $p_{A B}$ the price for supplying only $A$, only $B$, and both $A$ and $B$, respectively. One assumes that

$$
0<p_{B}<p_{A}<p_{A B}<p_{A}+p_{B} .
$$

City $A$ has several major clearwater springs, which makes this place a very well-known recreation place (trout fishing, kayak, ...). On the other hand, the springs can be easily collected. If $A$ decides to allow the pumping of its springs, the overall supply price will decrease dramatically. In this case, $A$ is said to be negative contributor. We denote by $q_{A}$ and $q_{A B}$ the price for supplying water to only $A$ and both $A$ and $B$, respectively, when $A$ is negative contributor. We have

$$
0<q_{A}<p_{A} \quad, \quad 0<q_{A B}<p_{A B} .
$$

We obtain the following game, assuming that the water consumption of city $C$ can only be provided by factory $F$ and that the marginal cost of $C$ is independent of the choices of cities $A$ and $B$.

$$
\begin{aligned}
& v(\emptyset, \emptyset)=0, \quad v(A, \emptyset)=p_{A}, \quad v(B, \emptyset)=p_{B}, \quad v(C, \emptyset)=p_{C} \\
& v(A B, \emptyset)=p_{A B}, \quad v(A C, \emptyset)=p_{A}+p_{C}, \quad v(B C, \emptyset)=p_{B}+p_{C} \\
& v(A B C, \emptyset)=p_{A B}+p_{C}, \quad v(\emptyset, A)=q_{A} \\
& v(B, A)=q_{A B}, \quad v(C, A)=q_{A}+p_{C}, \quad v(B C, A)=q_{A B}+p_{C} .
\end{aligned}
$$

Since the cost of supplying cities $A$ and $B$ on top of city $C$ is very high, this extra cost shall not be shared uniformly by all cities but shall be sponsored mainly by cities $A$ and $B$. This is a classical argument in cost sharing problems.

Now we see that a new problem arises here. If city $A$ produces enough water for itself and city $B$, then the cost of transporting water to $A$ and $B$ becomes negligible. However, $A$ is not surely willing to pump a great quantity of clear water from the springs since this could decrease the recreational attractiveness of the city. So $A$ must analyze the two options of being positive or negative contributor.

Example 4 (Multi-valued Ternary voting games) In ternary voting games, $v(S, T)$ can take only two values: 1 when the motion is accepted and 0 if it is rejected. Yet in many voting situations, the motion is not just simply accepted or rejected. For a strong winning coalition of yes voters, the initial motion is adopted. However, for a weak winning coalition of yes voters, a negotiation between the yes and the no voters usually yields a modified motion which can be seen as a weak version of the initial one. This situation corresponds to bills with amendment. In this case, since the initial motion is not applied, an intermediate value between 0 and 1 is assigned to $v(S, T)$. 
Example 5 (Heating Costs) In a building, heating is performed through floor heating. Each resident can choose among three attitudes: normal heating (say $70^{\circ} \mathrm{F}$ ), cold (say $64^{\circ} \mathrm{F}$ ) and warm heating (say $76^{\circ} \mathrm{F}$ ). Due to the technology that is used, residents cannot change by themselves the type of heating. A company is in charge of this job once a year, following the demand of each resident. $N$ is the set of apartments. Let us denote by $C(S, T)$ the global annual cost of ensuring hot temperature to $S$, cold temperature to $T$ and normal temperature to the remaining residents. Set $v(S, T)=$ $C(S, T)-C(\emptyset, \emptyset)$. The value $C(\emptyset, \emptyset)$ is supposed to be known. Each resident pays a monthly cost corresponding to heating so that she is charged $\frac{1}{n} C(\emptyset, \emptyset)$ at the end of the year. In the situation given by the bi-coalition $(S, T)$, we want to know how to share the extra cost or surplus $v(S, T)$ among the residents. In this example, the bi-cooperative game $v$ is monotone.

We have described so far cooperative games that are constructed directly from a cooperative situation. Another classical situation arises when the TU game is derived from a strategic game.

Example 6 (Conversion from a game in normal form) Consider here two neighbor states that are part of a Union. The level of industrial production of a state $i$ is denoted by $s_{i}$. The higher $s_{i}$ the wealthier the state but the more pollution there is. Since the two states are neighbor, the pollution caused by one state affects the other one to a certain degree $\alpha \in(0,1)$. We assume that the effect of production on pollution can be represented by a square function. Hence the utility function of state 1 is $u_{1}\left(s_{1}, s_{2}\right)=s_{1}-\left(s_{1}+\alpha s_{2}\right)^{2}$. The best response of state 1 given the level $s_{2}$ of state 2 is $\overline{s_{1}}\left(s_{2}\right)=\frac{1}{2}-\alpha s_{2}$. Likewise $u_{2}\left(s_{1}, s_{2}\right)=s_{2}-\left(s_{2}+\alpha s_{1}\right)^{2}$ and the best response for state 2 is $\overline{s_{2}}\left(s_{1}\right)=\frac{1}{2}-\alpha s_{1}$.

Utility functions $u_{1}$ and $u_{2}$ describe a game in normal form. This game has exactly one Nash equilibrium characterized by values $s_{1}=s_{2}=s:=$ $\frac{1}{2(1+\alpha)}$. On the other hand, the social welfare of the Union of the two states is $u_{1}\left(s_{1}, s_{2}\right)+u_{2}\left(s_{1}, s_{2}\right)$. The levels of production that maximize the social welfare for both states are $s_{1}=s_{2}=s^{\star}:=\frac{1}{2(1+\alpha)^{2}}$. Level $s^{\star}$ is lower than the selfish strategies $s$. Hence the Union wishes to introduce tax in order to give incentive to the states to reduce their level to $s^{\star}$. However, the Union does not want to impose a tax that is a direct function of $s_{i}-s^{\star}$ for state $i$.

The Union defines three areas: the negative one when $s_{i} \leq \theta s^{\star}$ (with $\theta \in(0,1))$ for a state $i$ willing to privilege the quality of life over profit, the null one when $s_{i} \in\left(\theta s^{\star}, s^{\star}\right]$ for state $i$ that chooses to adopt roughly the social optimum, and the positive one when $s_{i}>s^{\star}$ for a state $i$ that privileges profit to pollution. A bi-coalition $(S, T)$ describes the attitude chosen by each player, where $S, T$ and $N \backslash(S \cup T)$ are the sets of states having chosen the positive, negative and null attitude respectively. One can then compute the Nash equilibrium of the two states for all possible bi-coalitions $(S, T)$, i.e. the optimum values $\hat{s}_{1}(S, T)$ and $\hat{s}_{2}(S, T)$ of $s_{1}$ and $s_{2}$ in this situation. This defines the bi-cooperative game $v$ as follows: $v(S, T)=P\left(\hat{s}_{1}(S, T), \hat{s}_{2}(S, T)\right)$ 
where $P\left(s_{1}, s_{2}\right):=\left(s_{1}+\alpha s_{2}\right)^{2}+\left(s_{2}+\alpha s_{1}\right)^{2}$ is the overall pollution in the two states caused by levels $s_{1}$ and $s_{2}$. Using the best response functions $\overline{s_{1}}$ and $\overline{s_{2}}$, one easily gets the following values since $\alpha, \theta \in(0,1)$.

$$
\begin{aligned}
& \hat{s}_{1}(\{1,2\}, \emptyset)=\hat{s}_{2}(\{1,2\}, \emptyset)=s=\frac{1}{2(1+\alpha)} \\
& \hat{s}_{1}(\{1\}, \emptyset)=\frac{1+\alpha+\alpha^{2}}{2(1+\alpha)^{2}} \quad, \quad \hat{s}_{2}(\{1\}, \emptyset)=s^{\star}=\frac{1}{2(1+\alpha)^{2}} \\
& \hat{s}_{1}(\{2\}, \emptyset)=s^{\star}=\frac{1}{2(1+\alpha)^{2}} \quad, \quad \hat{s}_{2}(\{2\}, \emptyset)=\frac{1+\alpha+\alpha^{2}}{2(1+\alpha)^{2}} \\
& \hat{s}_{1}(\emptyset, \emptyset)=\hat{s}_{2}(\emptyset, \emptyset)=s^{\star}=\frac{1}{2(1+\alpha)^{2}} \\
& \hat{s}_{1}(\emptyset,\{1\})=\frac{\theta}{2(1+\alpha)^{2}} \quad \hat{s}_{2}(\emptyset,\{1\})=s^{\star}=\frac{1}{2(1+\alpha)^{2}} \\
& \hat{s}_{1}(\emptyset,\{2\})=s^{\star}=\frac{1}{2(1+\alpha)^{2}} \quad, \quad \hat{s}_{2}(\emptyset,\{2\})=\frac{\theta}{2(1+\alpha)^{2}} \\
& \hat{s}_{1}(\{1\},\{2\})=\frac{1+(1-\theta) \alpha+\alpha^{2}}{2(1+\alpha)^{2}} \quad, \quad \hat{s}_{2}(\{1\},\{2\})=\frac{\theta}{2(1+\alpha)^{2}} \\
& \hat{s}_{1}(\{2\},\{1\})=\frac{\theta}{2(1+\alpha)^{2}} \quad, \quad \hat{s}_{2}(\{2\},\{1\})=\frac{1+(1-\theta) \alpha+\alpha^{2}}{2(1+\alpha)^{2}} \\
& \hat{s}_{1}(\emptyset,\{1,2\})=\hat{s}_{2}(\emptyset,\{1,2\})=\frac{\theta}{2(1+\alpha)^{2}}
\end{aligned}
$$

Bi-cooperative game $v$ depicts the overall level of pollution coming from both states 1 and 2. From a knowledge of $v$, the Union determines the contribution of each state to the overall pollution in any situation $(S, T)$. The tax imposed on each state is then the contribution of the state to pollution $v(S, T)$ times a constant factor. Based on that, each state will decide which level is best for itself.

\section{Definition of a value}

Let us try to define the notion of value for bi-cooperative games, in the spirit of what was done by Shapley for cooperative games [18].

Let us first take the point of view of a value, and denote by $\mathcal{G}(N)$ the set of cooperative games on $N$. In classical cooperative game theory, an imputation (more precisely, a pre-imputation) is a vector $x \in \mathbb{R}^{n}$ satisfying the efficiency principle, that is, $\sum_{i \in N} x_{i}=v(N)$ [4]. The imputation represents the share of the total worth of the game $v(N)$ among the players, assuming that all players have decided to join the grand coalition $N$. A value is a mapping $\phi: \mathcal{G}(N) \longrightarrow \mathbb{R}^{n}$ which assigns to every game an imputation. The well-known Shapley value [18] is defined by

$$
\phi_{i}^{\mathrm{Sh}}(v):=\sum_{S \subseteq N \backslash i} \frac{s !(n-s-1) !}{n !}[v(S \cup i)-v(S)] .
$$

In usual games, each player has only two options: either to join a coalition or to stay aside. Cost allocation problems [17,19] and multi-choice games [12] model situations in which each player has several levels of participation to the games.

In a cost allocation problem, each agent $i \in N$ is interested in one type of a personalized good (each agent asks for a special good) and may demand 
several items of a good. A demand vector $q \in \mathbb{N}^{n}$ describes the number of items asked by each agent. A cost function is a nondecreasing function $v: \mathbb{N}^{n} \rightarrow \mathbb{R}$ such that $v(0, \ldots, 0)=0$, where $v(q)$ is the overall cost for satisfying demand $q$. One is interested by cost-sharing methods that define for each agent $i \in N$ a nonnegative cost $x_{i}$ satisfying the budget balance condition $\sum_{i \in N} x_{i}=v(q)[17]$.

Unlike usual games where at the end, all players join the grand coalition (unless for games in coalition form), it is not assumed here that all players have decided to be positive contributors. In multi-choice games, the players are not necessarily participating to the game at the highest possible level. We denote hereafter by $S$ the set of players that have decided to be positive contributors, by $T$ the set of players that have decided to be negative contributors. The remaining players $N \backslash(S \cup T)$ are not participating to the game. In the situation depicted by bi-coalition $(S, T)$, the worth that is obtained by the players is $v(S, T)$. Let $\phi^{S, T}(v): \mathcal{G}^{[2]}(N) \rightarrow \mathbb{R}^{N}$ be the payoff vector in the situation of bi-coalition $(S, T)$ as described previously. We are interested in defining this share $\phi^{S, T}(v)$.

In the Weber characterization of the Shapley value, four axioms are used: linearity, null player, symmetry and efficiency [20]. Consider first linearity. This axiom states that if several games are combined linearly then the values of each individual game shall be combined in the same way to obtain the value of the resulting game. This axiom is trivially extended to the case of bi-cooperative games.

Linearity (Lin): $\phi^{S, T}$ is linear on $\mathcal{G}^{[2]}(N)$.

Proposition 1 Under Lin, for all $i \in N$, there exists real constants $a_{S^{\prime}, T^{\prime}}^{i, S, T}$ for all $\left(S^{\prime}, T^{\prime}\right) \in \mathcal{Q}(N)$ such that for every game $v \in \mathcal{G}^{[2]}(N)$

$$
\phi_{i}^{S, T}(v)=\sum_{\left(S^{\prime}, T^{\prime}\right) \in \mathcal{Q}(N)} a_{S^{\prime}, T^{\prime}}^{i, S, T} v\left(S^{\prime}, T^{\prime}\right) .
$$

The proof of the proposition and all other results are given in the appendix.

In the Weber characterization of the Shapley value [20], the second axiom is called null player. It says that if a player $i$ is null, i.e. $v\left(S^{\prime} \cup\{i\}\right)=v\left(S^{\prime}\right)$ for any $S^{\prime} \subseteq N \backslash\{i\}$, then this player does not contribute at all to any coalition and thus the payoff for this player shall be zero. For bi-cooperative games, a player is said to be null if the asset is exactly the same if she joins the positive or the negative contributors.

Definition 1 The player $i$ is said to be null for the bi-cooperative game $v$ if $v\left(S^{\prime}, T^{\prime} \cup\{i\}\right)=v\left(S^{\prime}, T^{\prime}\right)=v\left(S^{\prime} \cup\{i\}, T^{\prime}\right)$ for any $\left(S^{\prime}, T^{\prime}\right) \in \mathcal{Q}(N \backslash\{i\})$.

We propose the following axiom. 
Null player (Null): If a player $i$ is null for the bi-cooperative game $v \in \mathcal{G}^{[2]}(N)$ then $\phi_{i}^{S, T}(v)=0$.

Proposition 2 Under Lin and Null, there exists for all $i \in N, a_{S^{\prime}, T^{\prime}}^{i, S, T}$ for all $\left(S^{\prime}, T^{\prime}\right) \in \mathcal{Q}(N \backslash\{i\}), b_{S^{\prime}, T^{\prime}}^{i, S, T}$ for $\left(S^{\prime}, T^{\prime}\right) \in \mathcal{Q}(N \backslash\{i\})$ such that

$$
\begin{aligned}
\phi_{i}^{S, T}(v)= & \sum_{\left(S^{\prime}, T^{\prime}\right) \in \mathcal{Q}(N \backslash\{i\})} a_{S^{\prime}, T^{\prime}}^{i, S, T}\left[v\left(S^{\prime} \cup\{i\}, T^{\prime}\right)-v\left(S^{\prime}, T^{\prime}\right)\right] \\
& +\sum_{\left(S^{\prime}, T^{\prime}\right) \in \mathcal{Q}(N \backslash\{i\})} b_{S^{\prime}, T^{\prime}}^{i, S, T}\left[v\left(S^{\prime}, T^{\prime} \cup\{i\}\right)-v\left(S^{\prime}, T^{\prime}\right)\right] .
\end{aligned}
$$

It is possible to replace Null by a stronger axiom, namely the dummy player axiom. For a usual TU game $v$, a player $i$ is said to be dummy if $v\left(S^{\prime} \cup\{i\}\right)=v\left(S^{\prime}\right)+v(\{i\})$ for all $S^{\prime} \subseteq N \backslash\{i\}$. The contribution of this player to any coalition is constant. Thus the payoff for this player shall by his solo worth $v(\{i\})$. For a bi-cooperative game, a dummy player is a player whose contribution to any bi-coalition is always the same, whatever the (positive or negative) level of contribution. This implies in particular that this player is a symmetric contributor.

Definition 2 The player $i$ is said to be dummy for the bi-cooperative game $v$ if there exists $\lambda \in \mathbb{R}$ such that $v\left(S^{\prime} \cup\{i\}, T^{\prime}\right)-v\left(S^{\prime}, T^{\prime}\right)=v\left(S^{\prime}, T^{\prime} \cup\right.$ $\{i\})-v\left(S^{\prime}, T^{\prime}\right)=\lambda$ for any $\left(S^{\prime}, T^{\prime}\right) \in \mathcal{Q}(N \backslash\{i\})$. Constant $\lambda$ is called the constant added value of player $i$ to $v$.

Dummy player (Dum): If a player $i$ is dummy for the bi-cooperative game $v \in \mathcal{G}^{[2]}(N)$ then $\phi_{i}^{S, T}(v)=\lambda$, where $\lambda \in \mathbb{R}$ is the constant added value of player $i$ to $v$.

Proposition 3 Under Lin and Dum, there exists for all $i \in N, a_{S^{\prime}, T^{\prime}}^{i, S o r}$ all $\left(S^{\prime}, T^{\prime}\right) \in \mathcal{Q}(N \backslash\{i\}), b_{S^{\prime}, T^{\prime}}^{i, S}$ for $\left(S^{\prime}, T^{\prime}\right) \in \mathcal{Q}(N \backslash\{i\})$ such that (4) holds. Moreover,

$$
\sum_{\left(S^{\prime}, T^{\prime}\right) \in \mathcal{Q}(N \backslash\{i\})} a_{S^{\prime}, T^{\prime}}^{i, S, T}+\sum_{\left(S^{\prime}, T^{\prime}\right) \in \mathcal{Q}(N \backslash\{i\})} b_{S^{\prime}, T^{\prime}}^{i, S, T}=1 .
$$

Replacing Null by Dum in Proposition 2, one sees that the coefficients become normalized since they sum up to one.

The basic idea of monotonicity of solutions is that if the wealth that the players get collectively increases then the payoff of the players shall not decrease. Property called Aggregate Monotonicity (AM) states that if the worth for the grand coalition increases and the value of the game for sub-coalitions remains the same, then the payoff vector shall not decrease [15]. Clearly solutions are not monotone w.r.t. the game since, for instance, 
increasing the game on a coalition $S$ cannot imply increasingness of the payoff vector for players outside $S$. Hence, one shall be careful when giving a more general form of the aggregate monotonicity axiom.

We define monotonicity in the following way. Let $(N, v)$ and $\left(N, v^{\prime}\right)$ two TU games such that there exists $i \in N$ with $v^{\prime}(K)=v(K)$ and $v^{\prime}(K \cup\{i\}) \geq$ $v(K \cup\{i\})$ for all $K \subseteq N \backslash\{i\}$. Then the contribution of player $i$ to game $v^{\prime}$ is larger than that of player $i$ to game $v$. Then the payoff of $i$ in game $v$ shall not be greater to that of $i$ in $v^{\prime}$.

Generalizing that to bi-cooperative games, we obtain

Monotonicity (Mon): Let $v, v^{\prime}$ be two bi-cooperative games such that there exists $i \in N$ with

$$
\left\{\begin{array}{l}
v^{\prime}\left(S^{\prime}, T^{\prime}\right)=v\left(S^{\prime}, T^{\prime}\right) \\
v^{\prime}\left(S^{\prime} \cup\{i\}, T^{\prime}\right) \geq v\left(S^{\prime} \cup\{i\}, T^{\prime}\right) \\
v^{\prime}\left(S^{\prime}, T^{\prime} \cup\{i\}\right) \geq v\left(S^{\prime}, T^{\prime} \cup\{i\}\right)
\end{array}\right.
$$

for all $\left(S^{\prime}, T^{\prime}\right) \in \mathcal{Q}(N \backslash\{i\})$, then $\phi_{i}^{S, T}\left(v^{\prime}\right) \geq \phi_{i}^{S, T}(v)$.

When $i$ joins the positive coalition $S^{\prime}$, the added value for $i$ is larger for game $v^{\prime}$ than for $v$. When $i$ joins negative coalition $T^{\prime}$, the negative added value for $i$ is smaller in absolute value for $v^{\prime}$ than for $v$ (the decrease of the value of the game when $i$ joins the negative contributors is less important for $v^{\prime}$ than for $v$ ). Then the payoff of $i$ in $v$ shall not be greater to that of $i$ in $v^{\prime}$.

Proposition 4 Under Lin, Null and Mon, for all $i \in N$, there exists $a_{S^{\prime}, T^{\prime}}^{i, S, T} \geq 0$ for all $\left(S^{\prime}, T^{\prime}\right) \in \mathcal{Q}(N \backslash\{i\}), b_{S^{\prime}, T^{\prime}}^{i, S, T} \geq 0$ for $\left(S^{\prime}, T^{\prime}\right) \in \mathcal{Q}(N \backslash\{i\})$ such that

$$
\begin{aligned}
\phi_{i}^{S, T}(v)= & \sum_{\left(S^{\prime}, T^{\prime}\right) \in \mathcal{Q}(N \backslash\{i\})} a_{S^{\prime}, T^{\prime}}^{i, S, T}\left[v\left(S^{\prime} \cup\{i\}, T^{\prime}\right)-v\left(S^{\prime}, T^{\prime}\right)\right] \\
& +\sum_{\left(S^{\prime}, T^{\prime}\right) \in \mathcal{Q}(N \backslash\{i\})} b_{S^{\prime}, T^{\prime}}^{i, S, T}\left[v\left(S^{\prime}, T^{\prime} \cup\{i\}\right)-v\left(S^{\prime}, T^{\prime}\right)\right] .
\end{aligned}
$$

Replacing Null by Dum in Proposition 4, one would obtain, as for Proposition 3, that the non-negative coefficients $a^{i, S, T}$ and $b^{i, S, T}$ sum up to one. The probabilistic values [16] for bi-cooperative games are thus defined by Lin, Dum and Mon, and result from a variant of Proposition 4.

We finally introduce the efficiency axiom. $\phi^{S, T}(v)$ is a share of the worth obtained by the bi-coalition $(S, T)$.

Efficiency axiom (Eff): For every game in $\mathcal{G}^{[2]}(N)$,

$$
\sum_{i \in N} \phi_{i}^{S, T}(v)=v(S, T) .
$$


For $K \subseteq S \cup T$, we set

$$
V(K):=v(S \cap K, T \cap K)
$$

Proposition 5 Under Lin, Null, Mon and Eff, for all $i \in S \cup T$ there exists coefficients $a_{K}^{i, S, T} \geq 0$ for $K \subseteq(S \cup T) \backslash\{i\}$ such that

$$
\phi_{i}^{S, T}(v)=\sum_{K \subseteq(S \cup T) \backslash\{i\}} a_{K}^{i, S, T}[V(K \cup\{i\})-V(K)] .
$$

Moreover, if $i \in N \backslash(S \cup T), \phi_{i}^{S, T}(v)=0$

Replacing Null by Dum in Proposition 5, one would obtain as for Proposition 3 that the non-negative coefficients $a^{i, S, T}$ sum up to one. Quasivalues, which are defined as efficient probabilistic values [16], are obtained from Lin, Dum, Mon and Eff, and result from a variant of Proposition 5.

Proposition 5 is essential since it proves that the only terms of a bicooperative game $v$ that are used to determine $\phi^{S, T}(v)$ belong to

$$
\mathcal{Q}_{S, T}(N)=\left\{\left(S^{\prime}, T^{\prime}\right) \in \mathcal{Q}(N), S^{\prime} \subseteq S, T^{\prime} \subseteq T\right\}
$$

The payoff for a positive contributor depends only on her contribution from non-contribution to positive contributor. The information regarding how this player behaves when she becomes negative contributor is not relevant. The same argument can be used symmetrically for negative contributors. From Proposition 5, it is not possible to define an allocation rule that looks at the contribution for the opposite action to the one chosen by each agent (positive vs. negative contributor). Hence for the computation of the value, one can restrict the bi-cooperative game to a usual game (namely $V$ ). Even though we have much more information than what is contained in $V$, the remaining terms cannot be used in a coherent way. This argument justifies the use of classical cooperative game model.

This property holds for cost allocation problems. For such games, the value for a demand $q$ depends only on the restriction of the cost function on lower demands $\left\{q^{\prime} \in \mathbb{N}^{n}, \forall i \in N \quad q_{i}^{\prime} \leq q_{i}\right\}[19]$.

From Proposition 5, one easily gets the following corollary.

Corollary 1 We have

$$
\begin{aligned}
& \forall i \in N \backslash(S \cup T), \quad \phi_{i}^{S, T}(v)=0 \\
& \forall i \in S \text { with } i \text { positively monotone }, \quad \phi_{i}^{S, T}(v) \geq 0 \\
& \forall i \in T \text { with } i \text { negatively monotone }, \quad \phi_{i}^{S, T}(v) \leq 0
\end{aligned}
$$


If $i \in T$ is positively monotone and symmetric contributor, then $\phi_{i}^{S, T}(v) \geq$ 0 .

Let us give an interpretation of Corollary 1. For classical values, it is assumed that all players finally participate to the game so that the grand coalition is formed. If it is not the case, a coalition structure has formed. Then the players of each coalition share the worth obtained by that coalition [1]. In this case, the players that do not belong to the coalition get zero from this coalition. Hence it is natural that the payoff for a player that does not participate vanishes. Hence (6).

Inequality (7) comes from the fact that players of $S$ contribute positively to the game (provided $i$ is positively monotone) and deserve thus a nonnegative payoff. Likewise, inequality (8) holds since players of $T$ contribute negatively to the game (provided $i$ is negatively monotone) and deserve thus a non-positive payoff. In Example 1, (8) means that farmer 1 should be rewarded for having accepted that the pipes cut her field. In cost sharing problems, this condition is interpreted as a compensation for players that have accepted to be negative contributor, since this attitude causes them annoyance. The trouble caused by the choice to be negative contributor is clear in all three examples 1,3 and 5 .

Example 7 (Urban Water Supply continued) Let us give an interpretation of $(6),(7)$ and (8) in the context of Example 5. Value $\phi_{i}^{S, T}(v)$ corresponds to a share of the extra cost $v(S, T)$ compared to the cost $C(\emptyset, \emptyset)$ when all residents choice normal heating. The residents that asked for normal heating are not asked to pay more, which is natural since the overall cost would have been $C(\emptyset, \emptyset)$ if all residents had made this choice. The residents that asked for warm heating are charged extra-costs, and residents that asked for cold heating are paid back to some extent.

In the Weber characterization of the Shapley value [20], the last axiom that we have not considered yet is symmetry with respect to the players. It states that the rule for computing the share does not depend on the numbering of the players. It means that nothing special is done to one player compared to another one. In other words, the players are anonymous. This depicts fairness.

Let $\sigma$ be a permutation on $N$. With some abuse of notation, we denote $\sigma(K):=\{\sigma(i)\}_{i \in K}$. The role of the players of $S, T$ and $N \backslash(S \cup T)$ is different. So, for bi-cooperative games, symmetry holds only among players of $S$, players of $T$ and players of $N \backslash(S \cup T)$.

Intra-Coalition Symmetry axiom (IntraSym): For any permutation $\sigma$ of $N$ such that $\sigma(S)=S$ and $\sigma(T)=T$,

$$
\phi_{\sigma(i)}^{S, T}\left(v \circ \sigma^{-1}\right)=\phi_{i}^{S, T}(v) .
$$


Proposition 6 Under Lin, Null, Mon, Eff and IntraSym, there exists coefficients $p_{a, b}^{+, S, T} \geq 0$ for $a \in\{0, \ldots, s-1\}, b \in\{0, \ldots, t\}$, and there exists coefficients $p_{a, b}^{-, S, T} \geq 0$ for $a \in\{0, \ldots, s\}, b \in\{0, \ldots, t-1\}$ such that for all $i \in S \cup T$

$$
\phi_{i}^{S, T}(v)=\sum_{K \subseteq(S \cup T) \backslash\{i\}} p_{|K \cap S|,|K \cap T|}^{\epsilon_{i}, S, T}[V(K \cup\{i\})-V(K)] .
$$

where $\epsilon_{i}=$ "+" if $i \in S$ and $\epsilon_{i}=$ "-" if $i \in T$.

Inter-Coalition Symmetry axiom (InterSym): Let $i \in S$ and $j \in T$, and $v_{i}, v_{j}$ be two bi-cooperative games such that for all $\left(S^{\prime}, T^{\prime}\right) \in \mathcal{Q}((S \cup T) \backslash\{i, j\})$

$$
\begin{gathered}
v_{i}\left(S^{\prime} \cup\{i\}, T^{\prime}\right)-v_{i}\left(S^{\prime}, T^{\prime}\right)=v_{j}\left(S^{\prime}, T^{\prime}\right)-v_{j}\left(S^{\prime}, T^{\prime} \cup\{j\}\right) \\
v_{i}\left(S^{\prime} \cup\{i\}, T^{\prime} \cup\{j\}\right)-v_{i}\left(S^{\prime}, T^{\prime} \cup\{j\}\right) \\
=v_{j}\left(S^{\prime} \cup\{i\}, T^{\prime}\right)-v_{j}\left(S^{\prime} \cup\{i\}, T^{\prime} \cup\{j\}\right)
\end{gathered}
$$

Then

$$
\phi_{i}^{S, T}\left(v_{i}\right)=-\phi_{j}^{S, T}\left(v_{j}\right) .
$$

The axiom says that when the contribution of a player $i \in S$ to a game $v_{i}$ is exactly the opposite of that of a player $j \in T$ to a game $v_{j}$, then the incentive payoff for $i$ shall be exactly the opposite of the payoff for $j$.

Proposition 7 Under axioms Lin, Null, Mon, Eff, IntraSym and InterSym, there exists coefficients $p_{k}^{S, T} \geq 0$ for $k \in\{0, \ldots, s+t-1\}$,

$$
\phi_{i}^{S, T}(v)=\sum_{K \subseteq(S \cup T) \backslash\{i\}} p_{k}^{S, T}[V(K \cup\{i\})-V(K)] .
$$

Theorem $1 \phi^{S, T}$ satisfies to axioms Lin, Null, Mon, IntraSym, InterSym and Eff if and only if one has for all $i \in N$

$$
\phi_{i}^{S, T}(v)=\sum_{K \subseteq(S \cup T) \backslash\{i\}} \frac{k !(s+t-k-1) !}{(s+t) !}[V(K \cup\{i\})-V(K)] .
$$

The indices in $\phi_{i}^{S, T}(v)$ coincide with the Shapley value of cooperative game $V$ (see $(3))$.

Let us give now some properties of this value.

Property 1 If all players of $T$ are null, then for all $i \in S, \phi_{i}^{S, T}(v)$ equals the usual Shapley value $\phi_{i}^{S}(w)$ of player $i$ for game $(S, w)$, where $w\left(S^{\prime}\right):=$ $v\left(S^{\prime}, \emptyset\right), \forall S^{\prime} \subseteq S$.

Property 2 If all players of $S$ are null, then for $i \in T, \phi_{i}^{S, T}(v)$ equals the usual Shapley value $\phi_{i}^{T}(w)$ of player $i$ for game $(T, w)$, where $w\left(T^{\prime}\right):=v\left(\emptyset, T^{\prime}\right)$, $\forall T^{\prime} \subseteq T$. 
Combining previous results with Lin, we obtain the following property.

Property 3 If $v$ is decomposable (i.e. $v\left(S^{\prime}, T^{\prime}\right)=w_{+}\left(S^{\prime}\right)-w_{-}\left(T^{\prime}\right)$ for all $\left(S^{\prime}, T^{\prime}\right) \in \mathcal{Q}_{S, T}(N)$, where $w_{+}$and $w_{-}$are TU games) then

$$
\phi_{i}^{S, T}(v)=\left\{\begin{array}{l}
\phi_{i}^{S}\left(w_{+}\right) \quad \text { if } i \in S \\
-\phi_{i}^{T}\left(w_{-}\right) \quad \text { if } i \in T \\
0 \quad \text { otherwise }
\end{array}\right.
$$

\section{Mean prospect to a player}

One interesting problem is to know the mean prospect of player $i$ without the prior knowledge about the other players' intention. In other words, one does not know in advance coalitions $S$ and $T$. Two options are basically possible. The first one is to define the mean prospect to player $i$ considering all possible choices $(S, T)$ of the other players. If player $i$ chooses to be positive contributor, the mean payoff is

$$
\phi_{i}^{+}(v)=\frac{1}{3^{n-1}} \sum_{(S, T) \in \mathcal{Q}(N \backslash\{i\})} \phi_{i}^{S \cup\{i\}, T}(v)
$$

and the mean payoff to player $i$ is she chooses to be negative contributor is

$$
\phi_{i}^{-}(v)=\frac{1}{3^{n-1}} \sum_{(S, T) \in \mathcal{Q}(N \backslash\{i\})} \phi_{i}^{S, T \cup\{i\}}(v)
$$

The mean payoff of a player that chooses to be absent is always zero by definition. Finally the mean prospect to player $i$ if she did not make up her mind yet is

$$
\phi_{i}(v)=\frac{1}{3}\left(\phi_{i}^{+}(v)+\phi_{i}^{-}(v)\right)
$$

The second option is to compute the mean prospect not over all bicoalitions $(S, T)$ but over the bi-coalitions that form a partition of $N$ (i.e. $S \cup T=N)$. The idea is that the only relevant decisions we are interested in are positive and negative contributors. So each player is either positive or negative contributor. In the previous approach, none of the three decisions is ruled out. The reason is that non-participation is a very special situation, often not considered as a relevant option. In usual cooperative game theory, all players are supposed to participate at the end to the game. In our context, this means that all players will have a non zero level of participation to the game.

If player $i$ chooses to be positive contributor, the mean payoff is

$$
\phi_{i}^{+}(v)=\frac{1}{2^{n-1}} \sum_{S \subseteq N \backslash\{i\}} \phi_{i}^{S \cup\{i\}, N \backslash(S \cup\{i\})}(v) .
$$


One has by Theorem 1,

$$
\begin{aligned}
\phi_{i}^{+}(v)= & \frac{1}{2^{n-1}} \sum_{S \subseteq N \backslash\{i\}} \sum_{T \subseteq N \backslash\{i\}} \frac{t !(n-t-1) !}{n !} \\
& \times[v((T \cap S) \cup\{i\}, T \cap(N \backslash S))-v(T \cap S, T \cap(N \backslash S))] \\
= & \frac{1}{2^{n-1}} \sum_{(K, L) \in \mathcal{Q}(N \backslash\{i\})} d_{K, L}[v(K \cup\{i\}, L)-v(K, L)]
\end{aligned}
$$

where

$$
\begin{aligned}
d_{K, L} & =\sum_{S \subseteq N \backslash\{i\}} \sum_{\substack{T \subseteq N \backslash\{i\} \\
K=S \cap T, L=T \backslash S}} \frac{t !(n-t-1) !}{n !} \\
& =\frac{(k+l) !(n-k-l-1) !}{n !} \sum_{\substack{A \subseteq N \backslash(K \cup L \cup\{i\}) \\
S=K \cup A, T=K \cup L}} 1 \\
& =\frac{(k+l) !(n-k-l-1) !}{n !} \times 2^{n-1-k-l}
\end{aligned}
$$

Hence

$$
\phi_{i}^{+}(v)=\sum_{(K, L) \in \mathcal{Q}(N \backslash\{i\})} \frac{(k+l) !(n-k-l-1) !}{2^{k+l} n !}(v(K \cup\{i\}, L)-v(K, L))
$$

Similarly the mean prospect when $i$ decides to be negative contributor is

$$
\phi_{i}^{-}(v)=\sum_{(K, L) \in \mathcal{Q}(N \backslash\{i\})} \frac{(k+l) !(n-k-l-1) !}{2^{k+l} n !}(v(K, L \cup\{i\})-v(K, L))
$$

Finally, the mean prospect when $i$ has not yet made a decision (but will decide between positive and negative contributors) is

$$
\phi_{i}(v)=\frac{1}{2}\left(\phi_{i}^{+}(v)+\phi_{i}^{-}(v)\right) .
$$

\section{Discussion}

We investigate in this section the relevance of our proposal on previous examples.

Example 8 (Irrigation Network continued) Let us consider the bi-cooperative game given in Example 2. When farmer 1 does not allow the pipes to cut her field (positive contributor), the cost share of the irrigation network is :

$$
\phi_{1}^{\{1,2,3\}, \emptyset}(v)=\frac{1}{3} \quad, \quad \phi_{2}^{\{1,2,3\}, \emptyset}(v)=\phi_{3}^{\{1,2,3\}, \emptyset}(v)=\frac{47}{6} \approx 7.83
$$


The construction of the well is a necessary condition for any farmer to access water. Hence it is fair that the cost 1 of the well is divided equally among the farmers, hence a cost $1 / 3$ per farmer. Farmer 1 shall not pay more than her solo contribution to the well, since she does not need any pipe for her own usage of water. Hence the price for farmer 1 is $1 / 3$.

When farmer 1 decides to allow the pipes to cut her field (negative contributor), the cost share becomes

$\phi_{1}^{\{2,3\},\{1\}}(v)=-\frac{14}{3} \approx-4.66 \quad, \quad \phi_{2}^{\{2,3\},\{1\}}(v)=\phi_{3}^{\{2,3\},\{1\}}(v)=\frac{16}{3} \approx 5.33$.

We see that farmer 1 is given a significant amount of money as a compensation for allowing her field to be cut by the pipes. Hence farmer 1 earns $\phi_{1}^{\{1,2,3\}, \emptyset}(v)-\phi_{1}^{\{2,3\},\{1\}}(v)=5$ by switching her behavior from positive to negative contributor. One also notices that the switch of farmer 1 implies positive externalities on the two other players since the price they have to pay decreases from $\frac{47}{6}$ downto $\frac{16}{3}$.

In Example 3, city $A$ is actually the only city that has to make up its mind about which role to adopt. Cities $B$ and $C$ cannot become producer of water. For these cities, assigning a negative role to them is purely artificial and does not correspond to any reality. This example shows that in practice there are players for which one of the two roles is forbidden. Let $N^{+}$be the set of players that are allowed to be positive contributors, and $N^{-}$be the set of players that are allowed to be positive contributors. We assume that every player can choose to be absent from the game. In Example 3, one has $N^{+}=\{A, B, C\}$ and $N^{-}=\{A\}$. Let

$$
\mathcal{Q}_{N^{+}, N^{-}}(N):=\left\{(S, T) \in \mathcal{Q}(N), S \subseteq N^{+} \text {and } T \subseteq N^{-}\right\} .
$$

Let us denote by $v_{N^{+}, N^{-}}$a bi-cooperative game restricted to $\mathcal{Q}_{N^{+}, N^{-}}(N)$. By Proposition 5, the value $\phi^{S, T}(v)$ where $S \subseteq N^{+}$and $T \subseteq N^{-}$, requires information of $v$ only in $\mathcal{Q}_{N^{+}, N^{-}}(N)$ and thus of $v_{N^{+}, N^{-}}$. Hence our value is well-suited to the case of forbidden roles for players.

Example 9 (Urban Water Supply continued) Example 3 is a typical case of previous situation where cities $B$ and $C$ can only be positive contributors. One has

$$
\begin{array}{ll}
\phi_{A}^{A B C, \emptyset}(v)=\frac{1}{2}\left(p_{A}+p_{A B}-p_{B}\right), \quad \phi_{A}^{B C, A}(v)=\frac{1}{2}\left(q_{A}+q_{A B}-p_{B}\right), \\
\phi_{B}^{A B C, \emptyset}(v)=\frac{1}{2}\left(p_{B}+p_{A B}-p_{A}\right), \quad \phi_{B}^{B C, A}(v)=\frac{1}{2}\left(p_{B}+q_{A B}-q_{A}\right), \\
\phi_{C}^{A B C, \emptyset}(v)=\phi_{C}^{B C, A}(v)=p_{C} .
\end{array}
$$

Last relation comes from the fact that the contribution of city $C$ to the cost of any coalition is always $p_{C}$. By virtue of (1) and $(2), \phi_{A}^{A B C, \emptyset}(v)>$ 
$\phi_{B}^{A B C, \emptyset}(v)$ and $\phi_{A}^{B C, A}(v)<\phi_{A}^{A B C, \emptyset}(v)$. The benefit for $A$ when becoming negative contributor is $\frac{1}{2}\left(p_{A}+p_{A B}-q_{A}-q_{A B}\right)>0$. The decision by $A$ to allow pumping implies positive externalities on $B$ (and thus a decrease in the cost share for $B$ ) iff $q_{A B}-q_{A} \leq p_{A B}-p_{A}$. This condition means that the marginal cost for $B$ in the presence of $A$ is smaller when $A$ decides to be negative contributor instead of positive contributor.

Example 10 (Multi-valued Ternary voting games continued) For any $(S, T) \in$ $\mathcal{Q}(N), v(S, T)$ is the degree to which the initial motion has been adopted. It seems reasonable that $v$ is monotone. By virtue of Corollary 1 , one has $\phi_{i}^{S, T}(v) \geq 0$ for all $i \in S, \phi_{i}^{S, T}(v) \leq 0$ for all $i \in T, \phi_{i}^{S, T}(v)=0$ for all $i \in N \backslash(S \cup T)$. Value $\phi_{i}^{S, T}(v)$ can be interpreted as a power index. However, classical power indices are non-negative numbers. Here a negative value $\phi_{i}^{S, T}(v) \leq 0$ corresponds to a voter that defeats the motion, and can quantify the harmful power of that voter against the motion. One can define a power index in situation $(S, T)$ from $\phi_{i}^{S, T}(v)$ as $\psi_{i}^{S, T}(v)=\left|\phi_{i}^{S, T}(v)\right|$.

Reproducing what has been said in Section 4, the mean power index of voter $i$ when she defends the motion is

$$
\begin{aligned}
\psi_{i}^{+}(v) & =\frac{1}{2^{n-1}} \sum_{S \subseteq N \backslash\{i\}} \psi_{i}^{S \cup\{i\}, N \backslash(S \cup\{i\})}(v) \\
& =\sum_{(K, L) \in \mathcal{Q}(N \backslash\{i\})} \frac{(k+l) !(n-k-l-1) !}{2^{k+l} n !}(v(K \cup\{i\}, L)-v(K, L))
\end{aligned}
$$

Similarly, the mean power index of voter $i$ when she defeats the motion is

$$
\begin{aligned}
\psi_{i}^{-}(v) & =\frac{1}{2^{n-1}} \sum_{S \subseteq N \backslash\{i\}} \psi_{i}^{S, N \backslash S}(v) \\
& =\sum_{(K, L) \in \mathcal{Q}(N \backslash\{i\})} \frac{(k+l) !(n-k-l-1) !}{2^{k+l} n !}(v(K, L)-v(K, L \cup\{i\}))
\end{aligned}
$$

Hence the mean power index of voter $i$ is

$$
\begin{aligned}
& \psi_{i}(v)=\frac{1}{2}\left(\psi_{i}^{+}(v)+\psi_{i}^{-}(v)\right) \\
& =\sum_{(K, L) \in \mathcal{Q}(N \backslash\{i\})} \frac{(k+l) !(n-k-l-1) !}{2^{k+l} n !}(v(K \cup\{i\}, L)-v(K, L \cup\{i\}))
\end{aligned}
$$

Note that these indices do not sum up to 1 in the general case, which is also the case of some well-known power indices such as the Banzhaf one for usual voting games. 


\section{Comparison with previous works}

Let us compare these solutions to previous proposals.

The first proposal seems to be due to Felsenthal and Machover for ternary voting games [5]. We introduce it briefly, and for this we need some definitions. A ternary roll-call $R$ is a triplet $R=\left(\sigma_{R}, D_{R}, E_{R}\right)$ composed of an ordering $\sigma_{R}$ of the voters, a coalition $D_{R}$ which contains all voters that are in favor of the bill, and a coalition $E_{R}$ which contains all voters that are against the bill. The voters in $N \backslash\left(D_{R} \cup E_{R}\right)$ are abstentionist. The set of all ternary roll-calls for the voters $N$ is denoted by $\mathcal{T}_{N}$. When a voter $i$ is called he/she tells his/her opinion, that is to say in favor if $i \in D_{R}$, against if $i \in E_{R}$ or abstention otherwise. The pivot $\operatorname{Piv}(v, R)$ is the voter $\sigma_{R}(j)$ called at position $j$, where $j$ is the smallest index such that the result of the vote does not change after. The following definition is then proposed [5] :

$$
\phi_{i}^{\mathrm{FM}}(v)=\frac{\left|\left\{R \in \mathcal{T}_{N}, i=\operatorname{Piv}(v, R)\right\}\right|}{\left|\mathcal{T}_{N}\right|}
$$

where $\left|\mathcal{T}_{N}\right|=3^{n} n$ !. After some tedious computations, it can be shown that $[13]$

$$
\begin{array}{r}
\phi_{i}^{\mathrm{FM}}(v)=\sum_{(S, T) \in \mathcal{Q}(N \backslash\{i\})}\left[\left(\eta_{s}+\frac{\eta_{t}}{2}\right)(v(S \cup\{i\}, T)-v(S, T))\right. \\
\left.-\left(\eta_{t}+\frac{\eta_{s}}{2}\right)(v(S, T \cup\{i\})-v(S, T))\right]
\end{array}
$$

with

$$
\eta_{t}=\frac{1}{3^{n} n !} \sum_{t^{\prime}=0}^{t} \frac{t^{\prime} !\left(t-t^{\prime}\right) !}{t !}\left(n-1-t+t^{\prime}\right) !\left(t-t^{\prime}\right) ! 3^{t-t^{\prime}}
$$

A second proposal was done by the authors in [7], which distinguishes the cases where $i$ is a positive contributor (denoted by $\phi^{+}$) or a negative contributor (denoted by $\phi^{-}$):

$$
\begin{aligned}
& \phi_{i}^{+}(v)=\sum_{S \in N \backslash i} \frac{(n-s-1) ! s !}{n !}[v(S \cup i, N \backslash(S \cup i))-v(S, N \backslash(S \cup i))] \\
& \phi_{i}^{-}(v)=\sum_{S \in N \backslash i} \frac{(n-s-1) ! s !}{n !}[v(S, N \backslash(S \cup i))-v(S, N \backslash S)] .
\end{aligned}
$$

A single value can be obtained putting $\phi_{i}^{\mathrm{LG}}(v):=\phi_{i}^{+}(v)+\phi_{i}^{-}(v)$.

The most recent proposal seems to be due to Bilbao et al. [2]. It reads

$$
\phi_{i}^{\mathrm{B}}(v)=\sum_{(S, T) \in \mathcal{Q}(N \backslash i)}\left[\overline { p } _ { ( S , T ) } ^ { i } \left(v(S \cup i, T)-v(S, T)+\underline{p}_{(S, T)}^{i}(v(S, T)-v(S, T \cup i)]\right.\right.
$$


with $\bar{p}_{(S, T)}^{i}, \underline{p}_{(S, T)}^{i}$ given by

$$
\begin{aligned}
& \bar{p}_{s, t}=\frac{(n+s-t) !(n+t-s-1) !}{(2 n) !} 2^{n-s-t} \\
& \underline{p}_{s, t}=\frac{(n+t-s) !(n+s-t-1) !}{(2 n) !} 2^{n-s-t} .
\end{aligned}
$$

Comparing with the mean prospect to a player (formulas (9) to (11)), all these values differ from our proposal. The meaning of the power index of Felsenthal and Machover is deeply rooted in voting theory, and its explicit expression in terms of $v$ is not simple. It nevertheless satisfies natural axioms such as linearity Lin, null player Null, the classical symmetry axiom (which is stronger than IntraSym), and $2 \phi_{i}^{\mathrm{FM}}$ satisfies an efficiency axiom Eff1 which is very similar to the classical one:

Efficiency 1 (Eff1): $\sum_{i \in N} \phi_{i}(v)=v(N, \emptyset)-v(\emptyset, N)$.

Imposing in addition to Lin, Null, the classical symmetry axiom, and Eff1, a fifth one expressing a symmetry between the positive and and the negative parts, one obtains the value $\phi^{\mathrm{LG}}[13]$. An interesting feature of this value is that only a small subset of $\mathcal{Q}(N \backslash i)$ is used in the computation.

The proposal of Bilbao et al. also relies on Eff1. It is based on the following well known formula of the classical Shapley value:

$$
\phi_{i}(v)=\sum_{S \subseteq N, S \ni i} \frac{c(S \backslash i) c([S, N])}{n !}[v(S)-v(S \backslash i)]
$$

with $c([T, S])$ the number of maximal chains from $T$ to $S, c(S):=c([\emptyset, S])$, $c([S, S])=c(\emptyset)=1$. In fact, the coefficients $\bar{p}_{s, t}, \underline{p}_{s, t}$ are the relative numbers of maximal chains from $(\emptyset, N)$ to $(N, \emptyset)$ passing by $(S, T),(S \cup i, T)$ and $(S, T \cup i),(S, T)$ respectively. Hence, $\phi^{\mathrm{B}}$ is a transposition of $(12)$ to $\mathcal{Q}(N)$.

An important remark is that Eff1 is satisfied by all previous proposals, while we use Eff instead. It turns out that the crucial point lies here. Axiom Eff1 implies that $\mathcal{Q}(N)$ has two remarkable elements which are $(N, \emptyset)$ and $(\emptyset, N)$. These are the top and bottom elements of $\mathcal{Q}(N)$ when the order relation is the one implied by monotonicity: $(S, T) \sqsubseteq\left(S^{\prime}, T^{\prime}\right)$ iff $S \subseteq S^{\prime}$ and $T \supseteq T^{\prime}$. This situation makes bi-cooperative games "isomorphic" to multichoice games with three levels of participation (say 0, 1 and 2), since $3^{N}$ endowed with the usual order on vectors is isomorphic to $(\mathcal{Q}(N), \sqsubseteq)$. To our opinion, bi-cooperative games are very different from 3-choice games.

By contrast, axiom Eff considers $(\emptyset, \emptyset)$ as a remarkable element in $\mathcal{Q}(N)$, as well as all elements $(S, N \backslash S), S \subseteq N$. Then the suitable order relation is now the product order, i.e., $(S, T) \subseteq\left(S^{\prime}, T^{\prime}\right)$ iff $S \subseteq S^{\prime}$ and $T \subseteq T^{\prime}$, making $(\emptyset, \emptyset)$ the bottom element, and all $(S, N \backslash S), S \subseteq N$ becoming top elements. We call this a bipolar extension of $2^{N}$, a concept which is studied in detail in $[9,8]$. This structure is much more in accordance with the meaning conveyed by bi-cooperative games. 


\section{Conclusion}

We have given examples that justify the use of an extension of TU games where each player that decides to participate to the game can choose between positive participation and negative participation. Our concern is then how to fairly share the wealth obtained by the players given their choice. Several axioms are defined to characterize a value. This value is based on the Shapley value. The positive contributors will receive a positive payoff whereas the negative contributors will receive a negative payoff.

The value $\phi$ is the sharing rule that the benevolent dictator will apply on the players. So if the game is a common knowledge, it can help the players in understanding what is their best choice, i.e., being positive or negative contributor. In cost sharing problems, the non-participation is not an option since all players want to access to the resource or the commons. In Example 1, farmer 1 has pros and cons for the two options. The difference $\phi_{1}^{\{1,2,3\}, \emptyset}(v)-\phi_{1}^{\{2,3\},\{1\}}(v)$ represents the financial gain for farmer 1 when switching from positive contribution to negative contribution. If this figure is larger than the estimated financial harm of allowing her parcel to be cut by a pipe, farmer 1 will accept to be negative contributor.

More generally, each player chooses her best response, given the strategy adopted by the other players. Hence rational players will certainly adopt a Nash-like equilibrium. This suggests that the coalitions $S$ and $T$ can be determined endogenously rather than given exogenously.

\section{References}

1. R. J. Aumann and J. H. Drèze. Cooperative games with coalition structures. Int. J. of Game Theory, 3:217-237, 1974.

2. J. M. Bilbao, J. R. Fernández, N. Jiménez, and J. J. López. The value for bi-cooperative games. Annals of Operations Research, to appear.

3. J. M. Bilbao, J. R. Fernandez, A. Jiménez Losada, and E. Lebrón. Bicooperative games. In J. M. Bilbao, editor, Cooperative games on combinatorial structures. Kluwer Acad. Publ., 2000.

4. T. Driessen. Cooperative Games, Solutions and Applications. Kluwer Academic Publishers, Dordrecht, 1988.

5. D. Felsenthal and M. Machover. Ternary voting games. Int. J. of Game Theory, 26:335-351, 1997.

6. M. Grabisch and Ch. Labreuche. Bi-capacities for decision making on bipolar scales. In EUROFUSE Workshop on Informations Systems, pages 185-190, Varenna, Italy, September 2002.

7. M. Grabisch and Ch. Labreuche. Bi-capacities. Part I: definition, Möbius transform and interaction. Fuzzy Sets and Systems, 151:211-236, 2005.

8. M. Grabisch and Ch. Labreuche. A general construction for unipolar and bipolar interpolative aggregation. In 4th Conf. of the Eur. Soc. for Fuzzy Logic And Technology (EUSFLAT) and 11th LFA Conf., pages 916-921, Barcelona, Spain, Sept. 2005. 
9. M. Grabisch and Ch. Labreuche. Bi-capacities: towards a generalization of Cumulative Prospect Theory. J. of Mathematical Psychology, submitted.

10. H.W. Norde H. Hamers, S. Miquel and S. van Velzen. Fixed tree game with repeated players. Discussion Paper Tilburg University, 87, 2003.

11. D. Henriet and H. Moulin. Traffic-based cost allocation in a network. Rand. J. Economy, 87:275-312, 1999.

12. C. R. Hsiao and T. E. S. Raghavan. Shapley value for multichoice cooperative games, I. Games and Economic Behavior, 5:240-256, 1993.

13. Ch. Labreuche and M. Grabisch. Axiomatization of the Shapley value and power index for bi-cooperative games. Technical report, CERMSEM, Université Paris I, 2006.

14. Ch. Labreuche and M. Grabisch. Generalized choquet-like aggregation functions for handling bipolar scales. Eur. J. of Operational Research, 172:931955, 2006.

15. N. Megiddo. On the monotonicity of the bargaining set, the kernel and the nucleolus of a game. SIAM J. on Applied Mathematics, 27:355-358, 1974.

16. D. Monderer and D. Samet. variations on the shapley value. In HandBook of Game Theory No. III (Ed. R.J. Aumann and S. Hart) - Chapter 54, 2001.

17. H. Moulin. Cooperative microeconomics. Princeton University Press, 1995.

18. L. S. Shapley. A value for $n$-person games. In H. W. Kuhn and A. W. Tucker, editors, Contributions to the Theory of Games, Vol. II, number 28 in Annals of Mathematics Studies, pages 307-317. Princeton University Press, 1953.

19. Y. Sprumont. Coherent cost-sharing rules. Games and Economic Behaviour, 33:126-144, 2000.

20. R. J. Weber. Probabilistic values for games. In A. E. Roth, editor, The Shapley Value. Essays in Honor of Lloyd S. Shapley, pages 101-119. Cambridge University Press, 1988.

\section{Appendix}

Proof of Proposition 1: Consider $\phi^{S, T}$ satisfying Lin. Let $U_{K, L}$ be defined by $U_{K, L}\left(S^{\prime}, T^{\prime}\right)=1$ if $S^{\prime}=K$ and $T^{\prime}=L, U_{K, L}\left(S^{\prime}, T^{\prime}\right)=0$ otherwise. Then we have $v=\sum_{(K, L) \in \mathcal{Q}(N)} v(K, L) U_{K, L}$. By Lin,

$$
\phi_{i}^{S, T}(v)=\sum_{(K, L) \in \mathcal{Q}(N)} v(K, L) \phi_{i}^{S, T}\left(U_{K, L}\right) .
$$

Setting $a_{K, L}^{i, S, T}:=\phi_{i}^{S, T}\left(U_{K, L}\right)$, we obtain the wished result.

Proof of Proposition 2: Consider $\left\{\phi_{i}^{S, T}(v)\right\}_{i \in N}$ satisfying Lin and Null.

By Proposition 1, there exists $c_{S^{\prime}, T^{\prime}}^{i, S,}$ for $\left(S^{\prime}, T^{\prime}\right) \in \mathcal{Q}(N)$ such that

$$
\phi_{i}^{S, T}(v)=\sum_{\left(S^{\prime}, T^{\prime}\right) \in \mathcal{Q}(N)} c_{S^{\prime}, T^{\prime}}^{i, S, T} v\left(S^{\prime}, T^{\prime}\right) .
$$


We write

$$
\begin{aligned}
\phi_{i}^{S, T}(v)= & \sum_{\left(S^{\prime}, T^{\prime}\right) \in \mathcal{Q}(N \backslash\{i\})} c_{S^{\prime}, T^{\prime}}^{i, S, T} v\left(S^{\prime}, T^{\prime}\right)+\sum_{\left(S^{\prime}, T^{\prime}\right) \in \mathcal{Q}(N \backslash\{i\})} c_{S^{\prime} \cup\{i\}, T^{\prime}}^{i, S, T} v\left(S^{\prime} \cup\{i\}, T^{\prime}\right) \\
& +\sum_{\left(S^{\prime}, T^{\prime}\right) \in \mathcal{Q}(N \backslash\{i\})} c_{S^{\prime}, T^{\prime} \cup\{i\}}^{i, S, T} v\left(S^{\prime}, T^{\prime} \cup\{i\}\right)
\end{aligned}
$$

Assume now that $i$ is null for the bi-cooperative game $v$. Hence

$$
\phi_{i}^{S, T}(v)=\sum_{\left(S^{\prime}, T^{\prime}\right) \in \mathcal{Q}(N \backslash\{i\})} v\left(S^{\prime}, T^{\prime}\right)\left[c_{S^{\prime}, T^{\prime}}^{i, S, T}+c_{S^{\prime} \cup\{i\}, T^{\prime}}^{i, S, T}+c_{S^{\prime}, T^{\prime} \cup\{i\}}^{i, S, T}\right] .
$$

This relation holds for any bi-cooperative game $v$ such that $i$ is null for $v$, in particular for all games satisfying for a given $(K, L) \in \mathcal{Q}(N \backslash i)$ :

$$
\forall\left(S^{\prime}, T^{\prime}\right) \in \mathcal{Q}(N \backslash i), \begin{cases}v\left(S^{\prime}, T^{\prime}\right) & =U_{K, L}\left(S^{\prime}, T^{\prime}\right) \\ v\left(S^{\prime} \cup i, T^{\prime}\right) & =v\left(S^{\prime}, T^{\prime}\right) \\ v\left(S^{\prime}, T^{\prime} \cup i\right) & =v\left(S^{\prime}, T^{\prime}\right) .\end{cases}
$$

By Null, this gives for all $\left(S^{\prime}, T^{\prime}\right) \in \mathcal{Q}(N \backslash\{i\})$

$$
c_{S^{\prime}, T^{\prime}}^{i, S, T}+c_{S^{\prime} \cup\{i\}, T^{\prime}}^{i, S, T}+c_{S^{\prime}, T^{\prime} \cup\{i\}}^{i, S, T}=0 .
$$

Consequently, formula (13) can be rearranged in such a way to give the wished form. Yet $c_{S^{\prime} \cup\{i\}, T^{\prime}}^{i, T}$ is denoted by $a_{S^{\prime}, T^{\prime}}^{i, S, T}$ and $c_{S^{\prime}, T^{\prime} \cup\{i\}}^{i, S, T}$ by $b_{S^{\prime}, T^{\prime}}^{i, S, T}$.

Proof of Proposition 3: Consider $\left\{\phi_{i}^{S, T}(v)\right\}_{i \in N}$ satisfying Lin and Dum. Axiom Dum implies Null. Hence by Proposition 2, there exists for all $i \in N, a_{S^{\prime}, T^{\prime}}^{i, S, T}$ for all $\left(S^{\prime}, T^{\prime}\right) \in \mathcal{Q}(N \backslash\{i\}), b_{S^{\prime}, T^{\prime}}^{i, S, T}$ for $\left(S^{\prime}, T^{\prime}\right) \in \mathcal{Q}(N \backslash\{i\})$ such that (4) holds. Assume that player $i$ is dummy. Then by Dum

$$
\sum_{\left(S^{\prime}, T^{\prime}\right) \in \mathcal{Q}(N \backslash\{i\})} a_{S^{\prime}, T^{\prime}}^{i, S, T} \lambda+\sum_{\left(S^{\prime}, T^{\prime}\right) \in \mathcal{Q}(N \backslash\{i\})} b_{S^{\prime}, T^{\prime}}^{i, S, T} \lambda=\lambda .
$$

This concludes the proof since one can take any $\lambda \in \mathbb{R}$.

Proof of Proposition 4: Let $i \in N$. Under Lin and Null,

$$
\begin{aligned}
\phi_{i}^{S, T}(v)= & \sum_{\left(S^{\prime}, T^{\prime}\right) \in \mathcal{Q}(N \backslash\{i\})} \alpha_{S^{\prime}, T^{\prime}}^{i, S,}\left[v\left(S^{\prime} \cup\{i\}, T^{\prime}\right)-v\left(S^{\prime}, T^{\prime}\right)\right] \\
& +\sum_{\left(S^{\prime}, T^{\prime}\right) \in \mathcal{Q}(N \backslash\{i\})} \beta_{S^{\prime}, T^{\prime}}^{i, S, T}\left[v\left(S^{\prime}, T^{\prime} \cup\{i\}\right)-v\left(S^{\prime}, T^{\prime}\right)\right] .
\end{aligned}
$$

Let $v$ be a monotone bi-cooperative game such that

$\min _{\left(S^{\prime}, T^{\prime}\right) \in \mathcal{Q}(N \backslash\{i\})} \min \left(v\left(S^{\prime} \cup\{i\}, T^{\prime}\right)-v\left(S^{\prime}, T^{\prime}\right), v\left(S^{\prime}, T^{\prime}\right)-v\left(S^{\prime}, T^{\prime} \cup\{i\}\right)\right)=: \delta>0$ 
Let $(K, L) \in \mathcal{Q}(N \backslash\{i\})$. Define $v^{\prime}$ as follows: For all $\left(S^{\prime}, T^{\prime}\right) \in \mathcal{Q}(N \backslash\{i\})$

$$
\left\{\begin{array}{l}
v^{\prime}\left(S^{\prime}, T^{\prime}\right)=v\left(S^{\prime}, T^{\prime}\right) \\
v^{\prime}\left(S^{\prime} \cup\{i\}, T^{\prime}\right)=\left\{\begin{array}{l}
v(K \cup\{i\}, L)+\frac{\delta}{2} \quad \text { if } S^{\prime}=K \text { and } T^{\prime}=L \\
v\left(S^{\prime} \cup\{i\}, T^{\prime}\right) \quad \text { otherwise }
\end{array}\right. \\
v^{\prime}\left(S^{\prime}, T^{\prime} \cup\{i\}\right)=v\left(S^{\prime}, T^{\prime} \cup\{i\}\right)
\end{array}\right.
$$

By definition of $\delta, v^{\prime}$ is monotone. Moreover

$$
\phi_{i}^{S, T}\left(v^{\prime}\right)-\phi_{i}^{S, T}(v)=\alpha_{K, L}^{i, S, T} \times \frac{\delta}{2}
$$

By Mon, we obtain $\alpha_{K, L}^{i, S, T} \geq 0$ for all $(K, L) \in \mathcal{Q}(N \backslash\{i\})$.

Define now $v^{\prime}$ by for all $\left(S^{\prime}, T^{\prime}\right) \in \mathcal{Q}(N \backslash\{i\})$

$$
\left\{\begin{array}{l}
v^{\prime}\left(S^{\prime}, T^{\prime}\right)=v\left(S^{\prime}, T^{\prime}\right) \\
v^{\prime}\left(S^{\prime} \cup\{i\}, T^{\prime}\right)=v\left(S^{\prime} \cup\{i\}, T^{\prime}\right) \\
v^{\prime}\left(S^{\prime}, T^{\prime} \cup\{i\}\right)=\left\{\begin{array}{l}
v(K, L \cup\{i\})+\frac{\delta}{2} \quad \text { if } S^{\prime}=K \text { and } T^{\prime}=L \\
v\left(S^{\prime}, T^{\prime} \cup\{i\}\right) \text { otherwise }
\end{array}\right.
\end{array}\right.
$$

$v^{\prime}$ is monotone. Hence

$$
\phi_{i}^{S, T}\left(v^{\prime}\right)-\phi_{i}^{S, T}(v)=\beta_{K, L}^{i, S, T} \times \frac{\delta}{2}
$$

By Mon, we obtain $\beta_{K, L}^{i, S, T} \geq 0$ for all $(K, L) \in \mathcal{Q}(N \backslash\{i\})$.

The proof of Proposition 5 is based on the following lemmas

Lemma 1 Under Lin, Null, Mon and Eff, one has for all $(K, L) \in \mathcal{Q}(N)$ with $(K, L) \notin\{(S, T),(\emptyset, \emptyset)\}$

$$
\sum_{i \in K} a_{K \backslash\{i\}, L}^{i, S, T}-\sum_{i \in L} b_{K, L \backslash\{i\}}^{i, S, T}-\sum_{i \in N \backslash(K \cup L)}\left(a_{K, L}^{i, S, T}-b_{K, L}^{i, S, T}\right)=0
$$

where the coefficients $a$ and $b$ are given by Proposition 4. 
Proof : Under Lin, Null and Mon, one has

$$
\begin{aligned}
& \sum_{i \in N} \phi_{i}^{S, T}(v)=\sum_{i \in N} \sum_{\left(S^{\prime}, T^{\prime}\right) \in \mathcal{Q}(N \backslash\{i\})} a_{S^{\prime}, T^{\prime}}^{i, S, T}\left[v\left(S^{\prime} \cup\{i\}, T^{\prime}\right)-v\left(S^{\prime}, T^{\prime}\right)\right] \\
& +\sum_{i \in N} \sum_{\left(S^{\prime}, T^{\prime}\right) \in \mathcal{Q}(N \backslash\{i\})} b_{S^{\prime}, T^{\prime}}^{i, S, T}\left[v\left(S^{\prime}, T^{\prime}\right)-v\left(S^{\prime}, T^{\prime} \cup\{i\}\right)\right] \\
& =\sum_{(K, L) \in \mathcal{Q}(N)} v(K, L) \times\left[\sum_{\substack{i \in N,\left(S^{\prime}, T^{\prime}\right) \in \mathcal{Q}(N \backslash\{i\}) \\
K=S^{\prime} \cup\{i\}, L=T^{\prime}}} a_{S^{\prime}, T^{\prime}}^{i, S, T}\right. \\
& \left.-\sum_{\substack{i \in N,\left(S^{\prime}, T^{\prime}\right) \in \mathcal{Q}(N \backslash\{i\}) \\
K=S^{\prime}, L=T^{\prime} \cup\{i\}}} b_{S^{\prime}, T^{\prime}}^{i, S, T}-\sum_{\substack { i \in N \\
\begin{subarray}{c}{\left(S^{\prime}, T^{\prime}\right) \in \mathcal{Q}(N \backslash\{i\}) \\
K=S^{\prime}, L=T^{\prime}{ i \in N \\
\begin{subarray} { c } { ( S ^ { \prime } , T ^ { \prime } ) \in \mathcal { Q } ( N \backslash \{ i \} ) \\
K = S ^ { \prime } , L = T ^ { \prime } } }\end{subarray}}\left(a_{S^{\prime}, T^{\prime}}^{i, S, T}-b_{S^{\prime}, T^{\prime}}^{i, S, T}\right)\right] \\
& =\sum_{(K, L) \in \mathcal{Q}(N)} v(K, L) \times\left[\sum_{i \in K} a_{K \backslash\{i\}, L}^{i, S, T}-\sum_{i \in L} b_{K, L \backslash\{i\}}^{i, S, T}\right. \\
& \left.-\sum_{i \in N \backslash(K \cup L)}\left(a_{K, L}^{i, S, T}-b_{K, L}^{i, S, T}\right)\right]
\end{aligned}
$$

Since this holds for all bi-cooperative game, the proof is completed by Eff.

Lemma 2 Consider coefficients $a_{K, L}^{i, S, T}$ and $b_{K, L}^{i, S, T}$ given by Proposition 4. For $K \subseteq N$ fixed, if the following two conditions are fulfilled

(i) (14) holds for all $L \subseteq N \backslash K$;

(ii) $a_{K, L}^{i, S, T}=0$ for all $L \subseteq N \backslash K$ and all $i \in N \backslash(K \cup L)$;

then

$$
\left\{\begin{array}{l}
\forall i \in K, \forall L \subseteq N \backslash K, \quad a_{K \backslash\{i\}, L}^{i, S, T}=0 \\
\forall L \subseteq N \backslash K, \forall i \in N \backslash(K \cup L), \quad b_{K, L}^{i, S, T}=0
\end{array}\right.
$$

Proof : By assumptions (i) and (ii),

$$
\begin{aligned}
0= & \sum_{L \subseteq N \backslash K}\left[\sum_{i \in K} a_{K \backslash\{i\}, L}^{i, S, T}-\sum_{i \in L} b_{K, L \backslash\{i\}}^{i, S, T}-\sum_{i \in N \backslash(K \cup L)}(\underbrace{a_{K, L}^{i, S, T}}_{=0}-b_{K, L}^{i, S, T})\right] \\
= & \sum_{L \subseteq N \backslash K} \sum_{i \in K} a_{K \backslash\{i\}, L}^{i, S, T}-\sum_{L^{\prime} \subseteq N \backslash K, i \in N \backslash\left(K \cup L^{\prime}\right)} b_{K, L^{\prime}}^{i, S, T} \\
& +\sum_{L \subseteq N \backslash K, i \in N \backslash(K \cup L)} b_{K, L}^{i, S, T} \\
= & \sum_{L \subseteq N \backslash K, i \in K} a_{K \backslash\{i\}, L}^{i, S, T}
\end{aligned}
$$


By Proposition 4, all terms $a_{K \backslash\{i\}, L}^{i, S, T}$ are non-negative. Hence we deduce that

$$
\forall i \in K, \forall L \subseteq N \backslash K \quad a_{K \backslash\{i\}, L}^{i, S, T}=0 .
$$

Hence (14) becomes

$$
\sum_{i \in L} b_{K, L \backslash\{i\}}^{i, S, T}-\sum_{i \in N \backslash(K \cup L)} b_{K, L}^{i, S, T}=0
$$

for all $L \subseteq N \backslash K$. Let us prove by induction on $l$ that for all $l \in\{0, \ldots, n-$ $k-1\}$ and all $L \subseteq N \backslash K$ with $|L|=l$,

$$
b_{K, L}^{i, S, T}=0, \forall i \in N \backslash(K \cup L) .
$$

By (16) for $L=N \backslash K$, we obtain $\sum_{i \in N \backslash K} b_{K, N \backslash(K \cup\{i\})}^{i, S, T}=0$. From Proposition 4, all terms $b_{K, N \backslash(K \cup\{i\})}^{i, S, T}$ are non-positive. Hence for all $L \subseteq N \backslash K$ with $|L|=n-k-1, b_{K, L}^{i, S, T}=0$ for $i \in N \backslash(K \cup L)$. The induction assumption is thus satisfied for $l=n-k-1$.

Assume that the induction assumption holds till index $l$. Consider thus $L \subseteq N \backslash K$ with $|L|=l$. By (16) and the induction assumption

$$
\sum_{i \in L} b_{K, L \backslash\{i\}}^{i, S, T}=0 .
$$

From Proposition 4, we conclude that the induction assumption also holds for index $l-1$.

Lemma 3 Assume that Lin, Null, Mon and Eff hold. Consider coefficients $a_{K, L}^{i, S, T}$ and $b_{K, L}^{i, S, T}$ given by Proposition 4. Assume that $(S, T) \neq(N, \emptyset)$. Then for all $i \in N$ and all $(K, L) \in \mathcal{Q}(N \backslash\{i\})$

$$
|K| \geq s \Rightarrow a_{K, L}^{i, S, T}=0 \quad, \quad|K|>s \Rightarrow b_{K, L}^{i, S, T}=0
$$

Moreover, for all $i \in N$ and all $(K, L) \in \mathcal{Q}(N \backslash\{i\})$

$$
\begin{array}{rll}
(|K|<s \text { and } K \cup\{i\} \nsubseteq S) & \Rightarrow & a_{K, L}^{i, S, T}=0 \\
(|K| \leq s \text { and } K \nsubseteq S) & \Rightarrow & b_{K, L}^{i, S, T}=0
\end{array}
$$

Proof : Assume that $(S, T) \neq(N, \emptyset)$. Let us show by induction on $|K|$ that for all $K \subseteq N$ with $|K|>s$, (15) holds. By Lemma 1, condition (i) of Lemma 2 is satisfied for all $K \subseteq N$ with $|K|>s$. Consider first the case $K=N$. Then condition (ii) in Lemma 2 is implicitly satisfied since it is void. By Lemma 2, then (15) holds for $K=N$. Assume now by induction that (15) is satisfied for all $K \subseteq N$ with $|K|=p \in\{s+2, \ldots, n\}$. Consider thus $K \subseteq N$ with $|K|=p-1$. By the induction assumption, condition (ii) 
holds. Hence by Lemma 2, (15) is fulfilled for $K$. We have thus shown the first part of the lemma.

We now show by induction on $p$ that for all $p \in\{s, \ldots, 0\}$, all $(K, L) \in$ $\mathcal{Q}(N)$ and all $i \in N \backslash(K \cup L)$

$$
\begin{array}{rll}
(|K|=p-1 \text { and } K \cup\{i\} \nsubseteq \subseteq S) & \Rightarrow & a_{K, L}^{i, S, T}=0 \\
(|K|=p \text { and } K \nsubseteq \subseteq S) & \Rightarrow & b_{K, L}^{i, S, T}=0
\end{array}
$$

For $K=S$, condition (i) of Lemma 2 is not fulfilled for $L=T$. Consider thus $K$ with $|K|=s$ and $K \neq S$. By the first part of the lemma, condition (ii) holds. By Lemma 1, (i) is also satisfied. Hence Lemma 2 can be applied, leading to the induction assumption for $p=s$. Assume now that the induction assumption holds at index $p$. Let $K$ with $|K|=p$ and $K \nsubseteq \subseteq S$. Since $K \nsubseteq S$, there does not exists $L \subseteq N \backslash K$ and $i \in N \backslash(K \cup L)$ such that $K \cup\{i\} \subseteq S$. Thus condition (ii) in Lemma 2 is satisfied by the induction assumption at $p$. By this lemma, we obtain the induction assumption at index $p-1$. This proves the second part of the lemma.

Lemma 4 Consider coefficients $a_{K, L}^{i, S, T}$ and $b_{K, L}^{i, S, T}$ given by Proposition 4. For $L \subseteq N$ fixed, if the following two conditions are fulfilled

(i) (14) holds for all $K \subseteq N \backslash L$;

(ii) $b_{K, L}^{i, S, T}=0$ for all $K \subseteq N \backslash L$ and all $i \in N \backslash(K \cup L)$;

then

$$
\left\{\begin{array}{l}
\forall i \in L, \forall K \subseteq N \backslash L, \quad b_{K, L \backslash\{i\}}^{i, S, T}=0 \\
\forall K \subseteq N \backslash L, \forall i \in N \backslash(K \cup L), \quad a_{K, L}^{i, S, T}=0
\end{array}\right.
$$

Proof : Similar to that of Lemma 2.

Lemma 5 Assume that Lin, Null, Mon and Eff hold. Consider coeffcients $a_{K, L}^{i, S, T}$ and $b_{K, L}^{i, S, T}$ given by Proposition 4. Assume that $(S, T) \neq(\emptyset, N)$. Then for all $i \in N$ and all $(K, L) \in \mathcal{Q}(N \backslash\{i\})$

$$
|L| \geq t \Rightarrow b_{K, L}^{i, S, T}=0 \quad, \quad|L|>t \Rightarrow a_{K, L}^{i, S, T}=0
$$

Moreover, for all $i \in N$ and all $(K, L) \in \mathcal{Q}(N \backslash\{i\})$

$$
\begin{array}{rll}
(|L|<t \text { and } L \cup\{i\} \nsubseteq T) & \Rightarrow & b_{K, L}^{i, S, T}=0 \\
(|L| \leq t \quad \text { and } L \nsubseteq T) & \Rightarrow & a_{K, L}^{i, S, T}=0
\end{array}
$$


Proof : Similar to that of Lemma 3.

We are now in position to show Proposition 5.

Proof of Proposition 5: $\phi^{S, T}$ has the expression given in Proposition 4. Let $i \in N$.

Let us show that for $(K, L) \in \mathcal{Q}(N \backslash\{i\})$

$$
\begin{aligned}
(K, L) & \notin\left\{\left(K^{\prime}, L^{\prime}\right) \in \mathcal{Q}(N \backslash\{i\}), K^{\prime} \cup\{i\} \subseteq S \text { and } L^{\prime} \subseteq T\right\} \\
& \Longrightarrow \quad a_{K, L}^{i, S, T}=0 \\
(K, L) & \notin\left\{\left(K^{\prime}, L^{\prime}\right) \in \mathcal{Q}(N \backslash\{i\}), K^{\prime} \subseteq S \text { and } L^{\prime} \cup\{i\} \subseteq T\right\} \\
\Longrightarrow \quad b_{K, L}^{i, S, T} & =0
\end{aligned}
$$

First of all, if $(S, T) \notin\{(N, \emptyset),(\emptyset, N)\}$, then by Lemmas 3 and 5 , for $(K, L) \in$ $\mathcal{Q}(N \backslash\{i\})$, one has $a_{K, L}^{i, S, T}=0$ if $K \cup\{i\} \nsubseteq S$ or $L \nsubseteq T$, and $b_{K, L}^{i, S, T}=0$ if $K \nsubseteq S$ or $L \cup\{i\} \nsubseteq T$. Hence (18) and (19) are proved.

Now when $(S, T)=(N, \emptyset),(18)$ and (19) follow from Lemma 5 . When $(S, T)=(\emptyset, N),(18)$ and $(19)$ follow from Lemma 3.

From (18) and (19), if $i \in N \backslash(S \cup T)$, all terms $a_{K, L}^{i, S, T}$ and $b_{K, L}^{i, S, T}$ vanish. Hence $\phi_{i}^{S, T}(v)=0$.

Let $i \in S$. Then by (18) and (19), all the $b_{K, L}^{i, S, T}$ terms vanish, and the only non-zero terms of $a_{K, L}^{i, S, T}$ are such that $K \subseteq S \backslash\{i\}$ and $L \subseteq T$. For $R \subseteq(S \backslash\{i\}) \cup T$, we set $c_{R}^{i, S, T}:=a_{R \cap S, R \cap T}^{i, S, T}$. Then we obtain

$$
\begin{aligned}
\phi_{i}^{S, T}(v) & =\sum_{R \subseteq(S \cup T) \backslash\{i\}} c_{R}^{i, S, T}[v((R \cap S) \cup\{i\}, R \cap T)-v(R \cap S, R \cap T)] \\
& =\sum_{R \subseteq(S \cup T) \backslash\{i\}} c_{R}^{i, S, T}[V(R \cup\{i\})-V(R)] .
\end{aligned}
$$

By Proposition $4, c_{R}^{i, S, T} \geq 0$.

Let $i \in T$. Then by (18) and (19), all the $a_{K, L}^{i, S, T}$ terms vanish, and the only non-zero terms of $b_{K, L}^{i, S, T}$ are such that $K \subseteq S$ and $L \subseteq T \backslash\{i\}$. For $R \subseteq S \cup(T \backslash\{i\})$, we set $c_{R}^{i, S, T}:=-b_{R \cap S, R \cap T}^{i, S, T}$. Then we obtain

$$
\begin{aligned}
\phi_{i}^{S, T}(v) & =\sum_{R \subseteq(S \cup T) \backslash\{i\}}-c_{R}^{i, S, T}[v(R \cap S, R \cap T)-v(R \cap S,(R \cap T) \cup\{i\})] \\
& =\sum_{R \subseteq(S \cup T) \backslash\{i\}} c_{R}^{i, S, T}[V(R \cup\{i\})-V(R)]
\end{aligned}
$$


By Proposition $4, c_{R}^{i, S, T} \geq 0$.

Proof of Proposition 6: By Lin, Null, Mon and Eff, we have

$$
\phi_{i}^{S, T}(v)=\sum_{K \subseteq(S \cup T) \backslash\{i\}} a_{K}^{i, S, T}[V(K \cup\{i\})-V(K)] .
$$

Moreover,

$$
\phi_{\sigma(i)}^{S, T}\left(v \circ \sigma^{-1}\right)=\sum_{K \subseteq(S \cup T) \backslash\{\sigma(i)\}} a_{K}^{\sigma(i), S, T}\left[V\left(\sigma^{-1}(K \cup\{\sigma(i)\})\right)-V\left(\sigma^{-1}(K)\right]\right.
$$

But $V\left(\sigma^{-1}(K \cup\{\sigma(i)\})\right)=V\left(\sigma^{-1}(K) \cup\{i\}\right)$. Hence

$$
\phi_{\sigma(i)}^{S, T}\left(v \circ \sigma^{-1}\right)=\sum_{K^{\prime} \subseteq(S \cup T) \backslash\{i\}} a_{\sigma\left(K^{\prime}\right)}^{\sigma(i), S, T}\left[V\left(K^{\prime} \cup\{i\}\right)-V\left(K^{\prime}\right)\right]
$$

where $K^{\prime}=\sigma^{-1}(K)$. By IntraSym, one obtains

$$
\forall K \subseteq(S \cup T) \backslash\{i\}, \quad a_{\sigma(K)}^{\sigma(i), S, T}=a_{K}^{i, S, T} .
$$

Consider first the case when $\sigma(i)=i$. Since $S$ and $T$ are invariant by $\sigma$, we conclude, for $i$ fixed, that $a_{K}^{i, S, T}$ depends only on the cardinality of $K \cap S$ and $K \cap T$. Hence

$$
a_{K}^{i, S, T}=: p_{|K \cap S|,|K \cap T|}^{i, S, T} .
$$

Now when $\sigma(i) \neq i$, we have thus

$$
p_{|K \cap S|,|K \cap T|}^{i, S, T}=a_{K}^{i, S, T}=a_{\sigma(K)}^{\sigma(i), S, T}=p_{|K \cap S|,|K \cap T|}^{\sigma(i), S, T} .
$$

We conclude that mapping $i \mapsto p_{|K \cap S|,|K \cap T|}^{i, S, T}$ depends only on whether $i \in S$ or $i \in T$.

Proof of Proposition 7: Under Lin, Null, Mon, Eff and IntraSym

$$
\phi_{i}^{S, T}(v)=\sum_{K \subseteq(S \cup T) \backslash\{i\}} p_{|K \cap S|,|K \cap T|}^{\epsilon_{i}, S, T}[V(K \cup\{i\})-V(K)] .
$$

Let $\delta v_{0}$ and $\delta v_{1}$ be any two bi-cooperative games on $(S \cup T) \backslash\{i, j\}$. Let $v_{i}$ and $v_{j}$ be two bi-cooperative games on $N$. Assume that $v_{i}$ can take any value on $\mathcal{Q}((S \cup T) \backslash\{i\})$. Then define $v_{i}$ on $\mathcal{Q}(S \cup T) \backslash \mathcal{Q}((S \cup T) \backslash\{i\})$ by

$$
v_{i}\left(S^{\prime} \cup\{i\}, T^{\prime}\right)=v_{i}\left(S^{\prime}, T^{\prime}\right)+\delta v_{\left|T^{\prime} \cap\{j\}\right|}\left(S^{\prime}, T^{\prime} \backslash\{j\}\right) .
$$

for all $\left(S^{\prime}, T^{\prime}\right) \in \mathcal{Q}((S \cup T) \backslash\{i\})$ Similarly $v_{j}$ can take any value on $\mathcal{Q}((S \cup$ $T) \backslash\{j\})$. Then define $v_{j}$ on $\mathcal{Q}(S \cup T) \backslash \mathcal{Q}((S \cup T) \backslash\{j\})$ by

$$
v_{j}\left(S^{\prime}, T^{\prime} \cup\{j\}\right)=v_{j}\left(S^{\prime}, T^{\prime}\right)-\delta v_{\left|S^{\prime} \cap\{i\}\right|}\left(S^{\prime} \backslash\{i\}, T^{\prime}\right) .
$$


for all $\left(S^{\prime}, T^{\prime}\right) \in \mathcal{Q}((S \cup T) \backslash\{j\})$ One has for all $\left(S^{\prime}, T^{\prime}\right) \in \mathcal{Q}((S \cup T) \backslash\{i, j\})$

$$
\begin{gathered}
v_{i}\left(S^{\prime} \cup\{i\}, T^{\prime}\right)-v_{i}\left(S^{\prime}, T^{\prime}\right)=\delta v_{0}\left(S^{\prime}, T^{\prime}\right)=v_{j}\left(S^{\prime}, T^{\prime}\right)-v_{j}\left(S^{\prime}, T^{\prime} \cup\{j\}\right) \\
v_{i}\left(S^{\prime} \cup\{i\}, T^{\prime} \cup\{j\}\right)-v_{i}\left(S^{\prime}, T^{\prime} \cup\{j\}\right) \\
=\delta v_{1}\left(S^{\prime}, T^{\prime}\right)=v_{j}\left(S^{\prime} \cup\{i\}, T^{\prime}\right)-v_{j}\left(S^{\prime} \cup\{i\}, T^{\prime} \cup\{j\}\right)
\end{gathered}
$$

One has

$$
\begin{aligned}
\phi_{i}^{S, T}\left(v_{i}\right) & =\sum_{K \subseteq(S \cup T) \backslash\{i, j\}} p_{|K \cap S|,|K \cap T|}^{+, S, T} \delta v_{0}(K \cap S, K \cap T) \\
& +\sum_{K \subseteq(S \cup T) \backslash\{i, j\}} p_{|K \cap S|,|K \cap T|+1}^{+, S, T} \delta v_{1}(K \cap S, K \cap T)
\end{aligned}
$$

and

$$
\begin{aligned}
\phi_{j}^{S, T}\left(v_{j}\right) & =-\sum_{K \subseteq(S \cup T) \backslash\{i, j\}} p_{|K \cap S|,|K \cap T|}^{-, S, T} \delta v_{0}(K \cap S, K \cap T) \\
& -\sum_{K \subseteq(S \cup T) \backslash\{i, j\}} p_{|K \cap S|+|,|,|K \cap T|}^{-, S} \delta v_{1}(K \cap S, K \cap T)
\end{aligned}
$$

Since this holds for all $\delta v_{0}$ and $\delta v_{1}$, we have, by InterSym, for all $a \in$ $\{0, \ldots, s-1\}, b \in\{0, \ldots, t-1\}$

$$
\begin{aligned}
& p_{a, b}^{+, S, T}=p_{a, b}^{-, S, T} \\
& p_{a, b+1}^{+, S, T}=p_{a+1, b}^{-, S, T}
\end{aligned}
$$

Thus if $a+b \leq s+t-2$

$$
p_{a, b}^{+, S, T}=p_{a, b}^{-, S, T}=p_{a-1, b+1}^{+, S, T}=\cdots=p_{1, a+b-1}^{-, S, T}=p_{0, a+b}^{+, S, T}
$$

We set thus

$$
p_{a+b}^{S, T}:=p_{a, b}^{+, S, T}=p_{a, b}^{-, S, T} .
$$

Moreover, we set for $a+b=s+t-1$

$$
p_{s+t-1}^{S, T}:=p_{s-1, t}^{+, S, T}=p_{s, t-1}^{-, S, T} .
$$

Hence for any $v$,

$$
\begin{aligned}
\phi_{i}^{S, T}(v) & =\sum_{K \subseteq(S \cup T) \backslash\{i\}} p_{|K \cap S|+|T \cap| K \cap T \mid}^{S, T}[V(K \cup\{i\})-V(K)] \\
& =\sum_{K \subseteq(S \cup T) \backslash\{i\}} p_{k}^{S, T}[V(K \cup\{i\})-V(K)]
\end{aligned}
$$

Proof of Theorem 1: The "if" part is straightforward and left to the reader. 
Under the above axioms,

$$
\begin{aligned}
\sum_{i \in N} \phi_{i}^{S, T}(v) & =\sum_{i \in N} \sum_{K \subseteq(S \cup T) \backslash\{i\}} p_{k}^{S, T}[V(K \cup\{i\})-V(K)] \\
& =\sum_{L \subseteq S \cup T} V(L) \times\left\{\sum_{\substack{i \in N, K \subseteq(S \cup T) \backslash\{i\} \\
L=K \cup\{i\}, i \in L}} p_{k}^{S, T}-\sum_{\substack{i \in N, \mathcal{K} \in(S \cup T) \backslash\{i\} \\
L=K, i \notin L}} p_{k}^{S, T}\right\} \\
& =\sum_{L \subseteq S \cup T} V(L) \times\left\{\sum_{\substack{i \in L \\
l, L}} p_{l-1}^{S, T}-\sum_{i \in(S \cup T) \backslash L} p_{l}^{S, T}\right\} \\
& =\sum_{L \subseteq S \cup T}\left[l \times p_{l-1}^{S, T}-(s+t-l) \times p_{l}^{S, T}\right] V(L)
\end{aligned}
$$

Axiom (E) implies that the coefficient of $V(S \cup T)$ should be 1, that of $V(\emptyset)$ should be -1 , and all others being 0 . This yields the following relations:

$$
\begin{aligned}
& (s+t) \times p_{s+t-1}^{S, T}=1 \\
& l \times p_{l-1}^{S, T}-(s+t-l) \times p_{l}^{S, T}=0 \quad \text { for all } l \in\{1, \ldots, s+t-1\}
\end{aligned}
$$

Hence

$$
p_{l}^{S, T}=\frac{l !(s+t-l-1) !}{(s+t) !} .
$$

Proof of Property 1: If all players of $T$ are null, then for $\left(S^{\prime}, T^{\prime}\right) \in$ $\mathcal{Q}_{S, T}(N), T^{\prime}=\left\{i_{1}, \ldots, i_{t^{\prime}}\right\}$

$$
\begin{aligned}
v\left(S^{\prime}, T^{\prime}\right) & =v\left(S^{\prime},\left\{i_{1}, \ldots, i_{t^{\prime}}\right\}\right)=v\left(S^{\prime},\left\{i_{1}, \ldots, i_{t^{\prime}-1}\right\}\right) \\
& =\cdots=v\left(S^{\prime},\left\{i_{1}\right\}\right)=v\left(S^{\prime}, \emptyset\right)=: w\left(S^{\prime}\right)
\end{aligned}
$$

